\title{
SPDES WITH COLOURED NOISE: ANALYTIC AND STOCHASTIC APPROACHES *,**
}

\author{
Marco Ferrante ${ }^{1}$ And Marta SAnZ-Solé ${ }^{2}$
}

\begin{abstract}
We study strictly parabolic stochastic partial differential equations on $\mathbb{R}^{d}, d \geq 1$, driven by a Gaussian noise white in time and coloured in space. Assuming that the coefficients of the differential operator are random, we give sufficient conditions on the correlation of the noise ensuring Hölder continuity for the trajectories of the solution of the equation. For self-adjoint operators with deterministic coefficients, the mild and weak formulation of the equation are related, deriving path properties of the solution to a parabolic Cauchy problem in evolution form.
\end{abstract}

Mathematics Subject Classification. 60H15, 60H25, 35R60.

Received February 22, 2005. Revised March 29, 2006.

\section{INTRODUCTION}

Stochastic partial differential equations (SPDEs) can be analized by different approaches related with the classical deterministic methods. Let us mention the variational point of view [13,20,21] and the semigroup approach [6], based on analytical methods, and the more genuine probabilistic setting using stochastic integration with respect to martingale measures $[5,28]$.

The variational approach leads in particular to a now very complete $L_{2}$-theory (see [21]). However, this theory does not provide with sharp results on the properties of the trajectories of the solutions of SPDEs, except in the time variable. A more deep analytical insight into parabolic SPDEs has been recently given by Krylov and Lototsky, developing an $L_{p}$-theory with $p \in[2, \infty)$ (see [14,15] and the references herein, [16]). This theory allows to obtain properties of the trajectories - both in time and space - quite sharp, using Sobolev type imbeddings. Let us point out that in $[14,15]$ the coefficients of the differential operator can be random, therefore the theory applies to a very general class of equations. In a similar spirit, parabolic SPDEs with deterministic coefficients in Hölder classes have been studied in [19].

In this paper we study stochastic partial differential equations in the whole space $\mathbb{R}^{d}$, with arbitrary dimension $d \geq 1$, driven by a Gaussian noise white in time and with homogeneous spatial correlation. The differential operator is strictly parabolic with random coefficients, the free terms are random as well. Using the analytical

Keywords and phrases. Stochastic partial differential equations, mild and weak solutions, random noise.

* The first author was partially supported by the grant COFIN 2001-015341-006 of MIUR, Italy.

** The second author was partially supported by the grant BFM2003-01345 from the Dirección General de Investigación, Ministerio de Ciencia y Tecnología, Spain.

1 Dipartimento di Matematica, Università di Padova, Via Belzoni 7, 35131 Padova, Italy; ferrante@math.unipd.it

2 Facultat de Matemàtiques, Universitat de Barcelona, Gran Via 585, 08007 Barcelona, Spain; marta.sanz@ub.edu

(c) EDP Sciences, SMAI 2006 
approach of [15] (see also [14]), we give sufficient conditions on the correlation of the noise ensuring the existence of a solution with values on some subspace of $L_{p}\left(\mathbb{R}^{d}\right), p \in[2, \infty)$, and then, by means of Sobolev type imbeddings, we obtain the existence of a random field, indexed by time and space, which is a version of the solution and has its trajectories jointly Hölder continuous in $t, x$.

A similar question using the evolution approach has been addressed in some previous articles. In fact, parabolic equations with random coefficients in spatial dimension $d=1$, driven by a space-time white noise have been studied in [3]. The main result is the existence of a continuous random field solution to the equation. The mild form of the equation contains a stochastic convolution with an anticipating integrand. Therefore, the analysis requires tools of anticipating stochastic calculus - a intricate machinery based on Malliavin calculus and needs a strong regularity in terms of the random component $\omega$. These type of hypothesis can be avoided with the analytic approach. In fact, in this situation stronger results are given in [15], Theorem 8.5 and Remark 8.7, where joint Hölder continuity is obtained.

For $d \geq 1$ and driving noise of the same kind that the one we are considering in this paper, joint Hölder continuity for the stochastic heat equation in its mild form has been obtained in [24]. The result, proved by means of Kolmogorov's continuity criterium, is an extension of the one stated in [28] for $d=1$.

The analytic approach considers the formal SPDE in a weak form (see (6), (7)). Studying the relationship between the weak and the mild formulation of the SPDE (see (24)) gives the possibility of transferring results obtained in the analytic setting to the evolution scenary. The last part of the article is devoted to this topic, in the particular case where the differential operator is self-adjoint and its coefficients are deterministic. In the framework of a $L_{2}$-theory, for a Neumann boundary-value problem with a strictly parabolic divergence operator, this question has been studied in [25].

\section{SOME PRELIMINARIES AND NOTATION}

We denote by $\mathcal{D}\left(\mathbb{R}^{d+1}\right)$ the space of Schwartz test functions [22] (p. 24). On a complete probability space $(\Omega, \mathcal{F}, P)$, we consider a Gaussian process $\left\{F(\phi), \phi \in \mathcal{D}\left(\mathbb{R}^{d+1}\right)\right\}$, mean zero, with covariance functional given by

$$
\begin{aligned}
E(F(\phi), F(\psi)) & =\int_{\mathbb{R}_{+}} \mathrm{d} s \int_{\mathbb{R}^{d}} \Gamma(\mathrm{d} x)(\phi(s, \cdot) * \widetilde{\psi}(s, \cdot))(x) \\
& =\int_{\mathbb{R}_{+}} \mathrm{d} s \int_{\mathbb{R}^{d}} \mu(\mathrm{d} \xi) \mathcal{F} \phi(s, \cdot)(\xi) \overline{\mathcal{F} \psi(s, \cdot)(\xi)} .
\end{aligned}
$$

In (1), $\Gamma$ is a non-negative, non-negative definite, tempered measure, $\widetilde{\psi}(s, x)=\psi(s,-x), \mu$ is the non-negative tempered measure on $\mathbb{R}^{d}$ defined by $\mathcal{F}^{-1} \Gamma$, where $\mathcal{F}$ denotes the Fourier transform operator. We notice that $\Gamma$ is a symmetric measure [22] (Chap. VII, Th. XVII).

For any test function $f, g \in \mathcal{D}\left(\mathbb{R}^{d}\right)$, the functional

$$
Q(f, g)=\int_{\mathbb{R}^{d}} \Gamma(\mathrm{d} x)(f * \widetilde{g})(x)
$$

is non-negative and translation invariant, that means, $Q(f, g)=Q\left(\tau_{x} f, \tau_{x} g\right)$, where $\tau_{x} f(\cdot)=f(\cdot+x)$ (see Gel'fand and Vilenkin [10], p. 169).

Following Dalang and Frangos [4] (see also Dalang [5]) the process $F$ can be extended to a worthy martingale measure in the sense of Walsh. We will denote by $\left\{F(t, A), t \geq 0, A \in \mathcal{B}_{b}\left(\mathbb{R}^{d}\right)\right\}$ this extension and by $\mathcal{F}_{t}$ the $\sigma$-field generated by $\left\{F(s, A), 0 \leq s \leq t, A \in \mathcal{B}_{b}\left(\mathbb{R}^{d}\right)\right\}$. 
Consider the inner product on $\mathcal{D}\left(\mathbb{R}^{d}\right)$ defined by

$$
\langle f, g\rangle_{\mathcal{H}}=\int_{\mathbb{R}^{d}} \Gamma(\mathrm{d} x)(f * \widetilde{g})(x) .
$$

Let $\mathcal{H}$ be the completion of $\mathcal{D}\left(\mathbb{R}^{d}\right)$ with respect to the norm derived from $\langle\cdot, \cdot\rangle_{\mathcal{H}}$. For any complete orthonormal system (CONS) $\left\{e_{j}, j \geq 0\right\} \subset \mathcal{D}\left(\mathbb{R}^{d}\right)$ of $\mathcal{H}$, define

$$
W^{k}(t)=\int_{0}^{t} \int_{\mathbb{R}^{d}} F(\mathrm{~d} s, \mathrm{~d} x) e_{k}(x),
$$

$k \geq 0$, where the integral must be understood in Walsh's sense. The process $\left\{W^{k}(t), t \in[0, T], k \geq 0\right\}$ is a sequence of independent standard Brownian motions.

One can check that for any predictable process $X$,

$$
\int_{0}^{t} \int_{\mathbb{R}^{d}} F(\mathrm{~d} s, \mathrm{~d} x) X(s, x)=\sum_{k=0}^{\infty} \int_{0}^{t} W^{k}(\mathrm{~d} s)\left\langle X(s, \cdot), e_{k}(\cdot)\right\rangle_{\mathcal{H}} .
$$

In particular, for any $\phi \in \mathcal{D}\left(\mathbb{R}^{d}\right)$

$$
F(t, \phi):=\int_{0}^{t} \int_{\mathbb{R}^{d}} F(\mathrm{~d} s, \mathrm{~d} x) \phi(x)=\sum_{k=0}^{\infty}\left\langle\phi, e_{k}\right\rangle_{\mathcal{H}} W^{k}(t) .
$$

Let $p \in(1,+\infty), n \in \mathbb{R}$ and $d \in \mathbb{N}$. We denote by $H_{p}^{n}=H_{p}^{n}\left(\mathbb{R}^{d}\right)$ the fractional Sobolev space consisting of distributions $g$ on $\mathbb{R}^{d}$ such that there exists $f \in L_{p}\left(\mathbb{R}^{d}\right)$ and $g=(1-\Delta)^{-\frac{n}{2}} f$. It is a Banach space endowed with the norm

$$
\|u\|_{n, p}=\left\|(1-\Delta)^{n / 2} u\right\|_{p}
$$

where $\|\cdot\|_{p}$ denotes the usual norm of $L_{p}\left(\mathbb{R}^{d}\right)$ and $\Delta$ is the Laplacian operator on $\mathbb{R}^{d}$. It is important to notice that $\|\cdot\|_{n, p} \leq\|\cdot\|_{m, p}$ for $n \leq m$; this gives rise to the embeddings

$$
\cdots \subset H_{p}^{m} \subset H_{p}^{n} \subset \cdots \subset L_{p} \subset \cdots \subset H_{p}^{-n} \subset H_{p}^{-m} \subset \cdots
$$

When $n \in \mathbb{Z}_{+}$, the spaces $H_{p}^{n}$ coincide with the classical Sobolev spaces $W_{p}^{n}$. Moreover, the space $\mathcal{C}_{0}^{\infty}$ of infinitely differentiable functions with compact support is dense in each $H_{p}^{n}$. We refer the reader to [2] and [27] for an extensive account on these spaces.

\section{SPDES WITH RANDOM COEFFICIENTS}

In this section, we analyze a parabolic SPDE, with Lipschitz coefficients, driven by a noise $F$ as has been described in Section 2, under the prespective of the general theory developed in [14,15]. More precisely, we exhibit a relationship between the covariance measure $\Gamma$ and a fractional differentiability degree $\eta$ leading, a.s., to jointly continuous solutions in time and in space.

The results might be considered as a complement of those in Section 8.3 in [15], where the spatial dimension is $d=1$ and the driving noise, white in time and in space. Their proof consists in showing that the assumptions of Theorem 5.1 in [15] (see also Th. 3.2 in [14]) are satisfied.

For the sake of completeness, we start by quoting some basic material from $[14,15]$.

Consider a fractional Sobolev space $H_{p}^{n}$, with fixed $p \in[2, \infty), n \in \mathbb{R}$. For any $u \in H_{p}^{n}, \phi \in \mathcal{C}_{0}^{\infty}$, we define

$$
(u, \phi)=\int_{\mathbb{R}^{d}}\left[(1-\Delta)^{n / 2} u\right](x)\left[(1-\Delta)^{-n / 2} \phi\right](x) \mathrm{d} x .
$$


Let $\tau$ be a stopping time with respect to $\left(\mathcal{F}_{t}\right)_{t \geq 0}$ and $\mathcal{P}$ be the predictable $\sigma$-field. Set $\mathbb{H}_{p}^{n}(\tau)=L_{p}\left(\rrbracket 0, \tau \rrbracket, \mathcal{P}, H_{p}^{n}\right)$, $\mathbb{H}_{p}^{n}:=\mathbb{H}_{p}^{n}(\infty)$. The spaces $\mathbb{H}_{p}^{n}(\tau)$ are a kind of stochastic fractional Sobolev spaces.

We also introduce the following notation:

$$
\begin{aligned}
& (f, g) \in \mathcal{F}_{p}^{n}(\tau) \text { if and only if } f \in \mathbb{H}_{p}^{n}(\tau), g \in \mathbb{H}_{p}^{n+1}\left(\tau, l^{2}\right), \text { and we set }\|(f, g)\|_{\mathcal{F}_{p}^{n}(\tau)}=\|f\|_{\mathbb{H}_{p}^{n}(\tau)}+ \\
& \|g\|_{\mathbb{H}_{p}^{n+1}\left(\tau, l^{2}\right)}
\end{aligned}
$$

where $\mathbb{H}_{p}^{n+1}\left(\tau, l^{2}\right)$ correspond to the space of square summable sequences of elements of $\mathbb{H}_{p}^{n+1}(\tau)$. We denote by $\left(w_{k}(t), t \in[0, T], k \geq 0\right)$ a sequence of independent standard Wiener processes.

Definition 1. (Def. 3.1 in [15].) For a distribution valued function $u \in \cap_{T>0} \mathbb{H}_{p}^{n}\left(\tau \wedge T\right.$ ), we write $u \in \mathcal{H}_{p}^{n}(\tau)$ if

$$
u_{x x} \in \mathbb{H}_{p}^{n-2}(\tau), u(0, \cdot) \in L_{p}\left(\Omega, \mathcal{F}_{0}, H_{p}^{n-2 / p}\right)
$$

and there exists $(f, g) \in \mathcal{F}_{p}^{n-2}(\tau)$ such that, for any $\phi \in \mathcal{C}_{0}^{\infty}$, the equality

$$
(u(t, \cdot), \phi)=(u(0, \cdot), \phi)+\int_{0}^{t} \mathrm{~d} s(f(s, \cdot), \phi)+\sum_{k=1}^{\infty} \int_{0}^{t} w^{k}(\mathrm{~d} s)\left(g^{k}(s, \cdot), \phi\right)
$$

holds for all $t \leq \tau$ a.s.

We set

$$
\|u\|_{\mathcal{H}_{p}^{n}(\tau)}=\left\|u_{x x}\right\|_{\mathbb{H}_{p}^{n-2}(\tau)}+\|(f, g)\|_{\mathcal{F}_{p}^{n-2}(\tau)}+\left(E\|u(0, \cdot)\|_{n-2 / p, p}^{p}\right)^{1 / p} .
$$

Let us recall the result on existence and uniqueness of solution for stochastic partial differential equations of parabolic type driven by a sequence of independent Wiener processes. First, we introduce some notation, then the assumptions and finally, the statement.

Fix $n \in \mathbb{R}$ and $\gamma \in[0,1[$ be such that $\gamma=0$ if $n=0, \pm 1, \pm 2, \ldots$; otherwise, $\gamma>0$ and is such that $|n|+\gamma$ is not an integer. Define

$$
B^{|n|+\gamma}= \begin{cases}B\left(\mathbb{R}^{d}\right) & \text { if } n=0 \\ \mathcal{C}^{|n|-1,1}\left(\mathbb{R}^{d}\right) & \text { if } n= \pm 1, \pm 2, \ldots \\ \mathcal{C}^{|n|+\gamma}\left(\mathbb{R}^{d}\right) & \text { otherwise }\end{cases}
$$

where $B\left(\mathbb{R}^{d}\right)$ is the Banach space of bounded functions on $\mathbb{R}^{d}, \mathcal{C}^{|n|-1,1}\left(\mathbb{R}^{d}\right)$ is the Banach space of $|n|-1$ times continuously differentiable functions whose derivatives of $(|n|-1)$-st order are Lipschitz; $\mathcal{C}^{|n|+\gamma}\left(\mathbb{R}^{d}\right)$ are Hölder spaces. The spaces $B^{|n|+\gamma}\left(l_{2}\right)$ are defined in the obvious way.

Consider the following equation on $\rrbracket 0, \tau \rrbracket$ :

$$
\mathrm{d} u(t, x)=\left[a^{i, j}(t, x) u_{x^{i}, x^{j}}(t, x)+f(t, x, u)\right] \mathrm{d} t+g^{k}(t, x, u) d w_{t}^{k} .
$$

Notice that, in comparison with equation (5.1) in Krylov [15], we take here $\sigma^{i k} \equiv 0$.

By a solution to the Cauchy problem for equation (6) with initial condition $u_{0}$, we mean a stochastic process $u \in \mathcal{H}_{p}^{n+2}(\tau)$ such that for any test function $\phi \in \mathcal{C}_{0}^{\infty}$,

$$
\begin{aligned}
(u(t, \cdot), \phi)= & (u(0, \cdot), \phi)+\int_{0}^{t} \mathrm{~d} s\left(a^{i, j}(s, \cdot) u_{x^{i}, x^{j}}(s, \cdot)+f(s, \cdot, u), \phi\right) \\
& +\int_{0}^{t} w^{k}(\mathrm{~d} s)\left(g^{k}(s, \cdot, u), \phi\right),
\end{aligned}
$$

for all $t \in \llbracket 0, \tau \rrbracket$. 
Let us introduce the following conditions on the differential operator and on the coefficients of the equation:

(A1): For any $i, j=1, \ldots, d$,

$$
a^{i, j}: \Omega \times \mathbb{R}_{+} \times \mathbb{R}^{d} \longrightarrow \mathbb{R}
$$

is $\mathcal{P} \otimes \mathcal{B}\left(\mathbb{R}^{d}\right)$ - measurable.

For any $\omega \in \Omega$ a.s. and $t \geq 0$, we have $a^{i, j}(t, \cdot) \in B^{|n|+\gamma}$ and $\left\|a^{i, j}(t, \cdot)\right\|_{B^{|n|+\gamma}} \leq K$, where $\gamma>0, n \notin \mathbb{Z}$ are such that $|n|+\gamma$ is not an integer.

Moreover, there exist $K, \delta>0$, such that for any $\omega \in \Omega, t \geq 0, x, \lambda \in \mathbb{R}^{d}$,

$$
\delta|\lambda|^{2} \leq a^{i, j}(t, x) \lambda^{i} \lambda^{j} \leq K|\lambda|^{2} .
$$

(A2): For any $u \in H_{p}^{n+2}, f(t, \cdot, u), g(t, \cdot, u)$ are predictable processes taking values in $H_{p}^{n}$ and $H_{p}^{n+1}\left(l_{2}\right)$, respectively.

In addition,

(1) $(f(\cdot, *, 0), g(\cdot, *, 0)) \in \mathcal{F}_{p}^{n}(\tau)$;

(2) $f, g$ are a.s. continuous in the third variable $u$;

(3) for any $\varepsilon>0$, there exists $K_{\varepsilon}$ such that for any $u, v \in H_{p}^{n+2}, t \geq 0$,

$$
\| f(t, \cdot, u)-f\left(t, \cdot, v\left\|_{n, p}+\right\| g(t, \cdot, u)-g(t, \cdot, v)\left\|_{n+1, p} \leq \varepsilon\right\| u-v\left\|_{n+2, p}+K_{\varepsilon}\right\| u-v \|_{n, p},\right.
$$

a.s.

The next result is a particular version of Theorem 5.1 in [15].

Theorem 2. Assume that (A1) and (A2) are satisfied. Let

$$
u_{0} \in L_{p}\left(\Omega, \mathcal{F}_{0}, H_{p}^{n+2-2 / p}\right) .
$$

Then the Cauchy problem (6) on $\rrbracket 0, \tau \rrbracket$ with initial condition $u(0, \cdot)=u_{0}$ has a unique solution $u \in \mathcal{H}_{p}^{n+2}(\tau)$. This solution satisfies

$$
\|u\|_{\mathcal{H}_{p}^{n+2}(\tau)} \leq N\left\{\|f(\cdot, *, 0)\|_{\mathbb{H}_{p}^{n}(\tau)}+\|g(\cdot, *, 0)\|_{\mathbb{H}_{p}^{n+1}\left(\tau, l_{2}\right)}+\left(E\left\|u_{0}\right\|_{n+2-2 / p, p}^{p}\right)^{1 / p}\right\},
$$

where the constant $N$ depends only on $d, n, \gamma, p, \delta, K, T$ and the function $K_{\varepsilon}$.

Consider now the equation

$$
\mathrm{d} u(t, x)=\left[a^{i, j}(t, x) u_{x^{i}, x^{j}}(t, x)+b^{i}(t, x) u_{x^{i}}(t, x)+f(t, x, u(t, x))\right] \mathrm{d} t+h(t, x, u(t, x)) F(\mathrm{~d} t, x),
$$

with initial condition $u(0, x)=u_{0}(x)$, where $t \in \mathbb{R}_{+}, x \in \mathbb{R}^{d}$ and $F$ is the Gaussian process introduced in the preceding section. The coefficients $f, h$ are random real functions defined on $\rrbracket 0, \tau \rrbracket \times \mathbb{R}^{d} \times \mathbb{R}$. Under suitable assumptions, we shall prove that this equation can be set in the framework of Theorem 2 and deduce Hölder continuity of the trajectories of its unique solution.

Let us write (8) into the form (6). We consider a CONS $\left\{e_{j}, j \geq 0\right\}$ of $\mathcal{H}$. We have,

$$
\begin{aligned}
\left\langle h(t, \cdot, u), e_{k}\right\rangle_{\mathcal{H}} & =\int_{\mathbb{R}^{d}} \Gamma(\mathrm{d} x)\left(h(t, \cdot, u) * \tilde{e}_{k}\right)(x) \\
& =\int_{\mathbb{R}^{d}} \mathrm{~d} y h(t, y, u) \int_{\mathbb{R}^{d}} \Gamma(\mathrm{d} x) \tilde{e}_{k}(x-y),
\end{aligned}
$$

where in the last equality we have applied Fubini's theorem. 
Therefore, the term $h(t, x, u(t, x)) F(\mathrm{~d} t, x)$ can be rewritten as $g^{k}(t, x, u(t, x)) W^{k}(\mathrm{~d} t)$ with

$$
g^{k}(t, x, u(t, x))=h(t, x, u(t, x)) \int_{\mathbb{R}^{d}} \Gamma(\mathrm{d} y) \tilde{e}_{k}(y-x),
$$

and $W^{k}$ defined in (2) (see (3)). Indeed, in the integral formulation, the contribution of the last term in (8) is, for $\phi \in \mathcal{C}_{0}^{\infty}, \int_{0}^{t} \int_{\mathbb{R}^{d}} F(\mathrm{~d} t, \mathrm{~d} x) \phi(x) h(t, x, u(t, x))$. By virtue of $(3)$ and $(9)$,

$$
\begin{aligned}
\int_{0}^{t} \int_{\mathbb{R}^{d}} F(\mathrm{~d} t, \mathrm{~d} x) \phi(x) h(t, x, u(t, x)) & =\sum_{k=0}^{\infty} \int_{0}^{t} W^{k}(\mathrm{~d} s)\left\langle h(t, \cdot, u(t, \cdot)) \phi, e_{k}\right\rangle_{\mathcal{H}} \\
& =\sum_{k=0}^{\infty} \int_{0}^{t} W^{k}(\mathrm{~d} s) \int_{\mathbb{R}^{d}} \mathrm{~d} y h(t, y, u(t, y)) \phi(y)\left(\int_{\mathbb{R}^{d}} \Gamma(\mathrm{d} x) \tilde{e}_{k}(x-y)\right) \\
& =\sum_{k=0}^{\infty} \int_{0}^{t} W^{k}(\mathrm{~d} s)\left(h(t, \cdot, u(t, \cdot)) \int_{\mathbb{R}^{d}} \Gamma(\mathrm{d} x) \tilde{e}_{k}(x-\cdot), \phi\right)_{2},
\end{aligned}
$$

where $(\cdot, \cdot)_{2}$ denotes the inner product in $L^{2}\left(\mathbb{R}^{d}\right)$.

Set

$$
v_{k}(x)=\int_{\mathbb{R}^{d}} \Gamma(\mathrm{d} y) \tilde{e}_{k}(y-x) .
$$

The following lemma provides a useful tool to apply Theorem 2 to equation (8), where $h(t, x, u(t, x)) F(\mathrm{~d} t, x)$ is replaced by $g^{k}(t, x, u(x, t)) W^{k}(\mathrm{~d} t)$.

For any $\eta>0$, we denote by $R_{\eta, d}(x)$ the kernel of the operator $(1-\Delta)^{-\eta / 2}$ on $\mathbb{R}^{d}$, that is,

$$
\left[(1-\Delta)^{-\eta / 2} u\right](x)=R_{\eta, d} * u .
$$

It is well known that

$$
R_{\eta, d}(x)=C_{\eta, d}|x|^{\frac{\eta-d}{2}} K_{\frac{d-\eta}{2}}(|x|)
$$

where $C_{\eta, d}$ is the reciprocal of $\pi^{d / 2} 2^{(d+\eta-2) / 2} \Gamma\left(\frac{\eta}{2}\right)$ and $K_{\nu}$ is the modified Bessel function of the third kind (see for instance [7] and also [18] for a more detailed presentation). Notice that $R_{\eta, d}(x)$ is a radial function and that

$$
R_{\eta_{1}, d} * R_{\eta_{2}, d}=R_{\eta_{1}+\eta_{2}, d}
$$

for any $\eta_{1}, \eta_{2}>0$. Hence,

$$
\nu_{\eta, d}:=\left\|R_{\eta, d}\right\|_{\mathcal{H}}^{2}=\int_{\mathbb{R}^{d}} \Gamma(\mathrm{d} x) R_{2 \eta, d}(x) .
$$

Lemma 3. Let $\eta \in(0, \infty), d \in \mathbb{N}$ be such that

$$
\nu_{\eta, d}=\left\|R_{\eta, d}\right\|_{\mathcal{H}}^{2}<\infty .
$$

Let $h \in L_{p}\left(\mathbb{R}^{d}\right), g^{k}=v_{k} h$. Then $g=\left\{g_{k}, k \geq 0\right\} \in H_{p}^{-\eta}\left(l_{2}\right)$ and

$$
\|g\|_{-\eta, p}=\|\bar{h}\|_{p} \leq C\|h\|_{p}
$$

with

and $C=\nu_{\eta, d}^{1 / 2}$

$$
\bar{h}(x)=\left\|R_{\eta, d}(x-\cdot) h\right\|_{\mathcal{H}}
$$


Proof. Fubini's theorem and Parseval's identity yield

$$
\begin{aligned}
\left\|(1-\Delta)^{-\eta / 2} g(x)\right\|_{l_{2}}^{2} & =\sum_{k=0}^{\infty}\left((1-\Delta)^{-\eta / 2} g^{k}(x)\right)^{2} \\
& =\sum_{k=0}^{\infty}\left(\left(R_{\eta, d} *\left(v_{k} h\right)\right)(x)\right)^{2} \\
& =\sum_{k=0}^{\infty}\left(\int_{\mathbb{R}^{d}} \mathrm{~d} y R_{\eta, d}(x-y)\left(\int_{\mathbb{R}^{d}} \Gamma(\mathrm{d} z) \tilde{e}_{k}(z-y)\right) h(y)\right)^{2} \\
& =\sum_{k=0}^{\infty}\left(\int_{\mathbb{R}^{d}} \Gamma(\mathrm{d} z)\left(\int_{\mathbb{R}^{d}} \mathrm{~d} y R_{\eta, d}(x-y) h(y) \tilde{e}_{k}(z-y)\right)\right)^{2} \\
& =\sum_{k=0}^{\infty}\left(\int_{\mathbb{R}^{d}} \Gamma(\mathrm{d} z)\left(R_{\eta, d}(x-\cdot) h * \tilde{e}_{k}\right)(z)\right)^{2} \\
& =\sum_{k=0}^{\infty}\left\langle R_{\eta, d}(x-\cdot) h, e_{k}\right\rangle_{\mathcal{H}}^{2}=\left\|R_{\eta, d}(x-\cdot) h\right\|_{\mathcal{H}}^{2} .
\end{aligned}
$$

Therefore,

$$
\begin{aligned}
\|g\|_{-\eta, p} & =\left\|(1-\Delta)^{-\eta / 2} g\right\|_{L_{p}\left(l_{2}\right)}=\left(\int_{\mathbb{R}^{d}} \mathrm{~d} x\left\|(1-\Delta)^{-\eta / 2} g(x)\right\|_{l_{2}}^{p}\right)^{1 / p} \\
& =\left(\int_{\mathbb{R}^{d}} \mathrm{~d} x\left\|R_{\eta, d}(x-\cdot) h\right\|_{\mathcal{H}}^{p}\right)^{1 / p}=\|\bar{h}\|_{p} .
\end{aligned}
$$

The second part of (12) is a consequence of Hölder's inequality. Indeed, first we notice that, since $\Gamma$ is translation invariant,

$$
\nu_{\eta, d}=\left\|R_{\eta, d}\right\|_{\mathcal{H}}^{2}=\left\|R_{\eta, d}(x-\cdot)\right\|_{\mathcal{H}}^{2} .
$$

Then,

$$
\begin{aligned}
\|\bar{h}\|_{p}^{p}= & \int_{\mathbb{R}^{d}} \mathrm{~d} x\left\|R_{\eta, d}(x-\cdot) h\right\|_{\mathcal{H}}^{p} \\
= & \int_{\mathbb{R}^{d}} \mathrm{~d} x\left(\int_{\mathbb{R}^{d}} \Gamma(\mathrm{d} y) \int_{\mathbb{R}^{d}} \mathrm{~d} z R_{\eta, d}(x-(y-z)) h(y-z) \widetilde{R}_{\eta, d}(x-z) \widetilde{h}(z)\right)^{\frac{p}{2}} \\
\leq & \int_{\mathbb{R}^{d}} \mathrm{~d} x\left(\left\|R_{\eta, d}(x-\cdot)\right\|_{\mathcal{H}}^{p-2}\right) \\
& \times\left[\int_{\mathbb{R}^{d}} \Gamma(\mathrm{d} y) \int_{\mathbb{R}^{d}} \mathrm{~d} z R_{\eta, d}(x-(y-z)) \widetilde{R}_{\eta, d}(x-z)|h(y-z) \widetilde{h}(z)|^{\frac{p}{2}}\right] .
\end{aligned}
$$


Thus, (13), Fubini's theorem and Schwarz's inequality and the invariance of Lebesgue measure imply

$$
\begin{aligned}
\|\bar{h}\|_{p}^{p} \leq & \nu_{\eta, d}^{\frac{p}{2}-1} \int_{\mathbb{R}^{d}} \mathrm{~d} x \int_{\mathbb{R}^{d}} \mathrm{~d} z \int_{\mathbb{R}^{d}} \Gamma(\mathrm{d} y) R_{\eta, d}(y-z) R_{\eta, d}(z) \\
& \times|h(y-z+x)|^{\frac{p}{2}}|\widetilde{h}(z-x)|^{\frac{p}{2}} \\
\leq & \nu_{\eta, d}^{\frac{p}{2}-1} \int_{\mathbb{R}^{d}} \Gamma(\mathrm{d} y)\left(R_{\eta, d} * \widetilde{R}_{\eta, d}\right)(y)\left(\int_{\mathbb{R}^{d}} \mathrm{~d} x|h(y-z+x)|^{p}\right)^{\frac{1}{2}} \\
& \times\left(\int_{\mathbb{R}^{d}} \mathrm{~d} x|\widetilde{h}(z-x)|^{p}\right)^{\frac{1}{2}} \\
= & \nu_{\eta, d}^{\frac{p}{2}}\|h\|_{p}^{p} .
\end{aligned}
$$

This completes the proof of the lemma.

Remark 4. Let $\Gamma(\mathrm{d} x)=\delta_{\{0\}}(x)$ and thus, $\|\cdot\|_{\mathcal{H}}=\|\cdot\|_{2}$. In this particular case (12) has been obtained in Lemma 8.4 of Krylov [15].

Proposition 4.4.1 in [18] establishes that, if

$$
\int_{\mathbb{R}^{d}} \frac{\mu(\mathrm{d} \xi)}{\left(1+|\xi|^{2}\right)^{\eta}}<+\infty
$$

then (11) holds true.

The behavior of the Bessel function $K_{\nu}$ is well-known (see for example [1] and also [18]). In fact, in a neighborhood $O^{+}$of 0 ,

$$
K_{\nu}(r) \sim \begin{cases}\log (r), & \text { if } \nu=0, \\ r^{-|\nu|}, & \text { if } \nu \neq 0 .\end{cases}
$$

While away from zero,

$$
K_{\nu}(r) \leq C_{\nu} e^{-r}
$$

This leads to the following conclusions, which have already appeared in previous discussions on different classes of SPDEs (for instance, in [23], [18]).

(1) Assume $0<\eta<\frac{d}{2}$. Then

$$
\nu_{\eta, d}<+\infty \Leftrightarrow \int_{O^{+}}|x|^{2 \eta-d} \Gamma(\mathrm{d} x)<+\infty
$$

(2) Let $\eta=\frac{d}{2}$. Then

$$
\nu_{\eta, d}<+\infty \Leftrightarrow \int_{O^{+}} \log \left(\frac{1}{|x|}\right) \Gamma(\mathrm{d} x)<+\infty .
$$

(3) If $\eta>\frac{d}{2}$. Then $\nu_{\eta, d}<+\infty$, without any additional condition on $\Gamma$.

Example 5 (Riesz kernels). Set $\Gamma(\mathrm{d} x)=|x|^{-\alpha} \mathrm{d} x$, with $\alpha \in(0, d)$. Then, for $\eta \in\left(0, \frac{d}{2}\right], \nu_{\eta, d}<+\infty$ if and only if $\alpha \in(0,2 \eta \wedge d)$.

Let us now introduce the set of hypotheses to be assumed in order to prove existence and uniqueness of solution for (8) and Hölder properties for its paths. Given $\gamma_{1}, \gamma_{2}>0$, we denote by $\mathcal{C}^{\gamma_{1}, \gamma_{2}}\left([0, t] \times \mathbb{R}^{d}\right)$, the space of real-valued functions defined on $[0, t] \times \mathbb{R}^{d}$, jointly Hölder continuous of order $\gamma_{1}$ in its first variable and $\gamma_{2}$ in its second one. 
(H1): For any $i, j=1, \cdots, n, a^{i, j}, b^{i}: \Omega \times \mathbb{R}_{+} \times \mathbb{R}^{d} \rightarrow \mathbb{R}$ are $\mathcal{P} \otimes \mathcal{B}\left(\mathbb{R}^{d}\right)$-measurable; there exists $\eta \in(0,1)$ such that, for any $\omega \in \Omega$ a.s. and $t \geq 0, a^{i, j}(\omega, t, \cdot) \in \mathcal{C}^{\alpha}\left(\mathbb{R}^{d}\right), \alpha \in(1+\eta, 2), b^{i}(\omega, t, \cdot) \in \mathcal{C}^{0,1}\left(\mathbb{R}^{d}\right)$, and

$$
\sup _{t \geq 0}\left[\|a(t, \cdot)\|_{\mathcal{C}^{\alpha}}+\|b(t, \cdot)\|_{\mathcal{C}^{0,1}}\right] \leq k .
$$

There exist $K, \delta>0$, such that for any $\omega \in \Omega$ a.s., $t \geq 0, x, \lambda \in \mathbb{R}^{d}$,

$$
\delta|\lambda|^{2} \leq \sum_{i, j=1}^{d} a^{i, j}(t, x) \lambda^{i} \lambda^{j} \leq K|\lambda|^{2} .
$$

(H2): $f, h: \Omega \times \mathbb{R}_{+} \times \mathbb{R}^{d} \times \mathbb{R} \rightarrow \mathbb{R}$ are such that, for any $x$ and $u, f(\cdot, x, u), h(\cdot, x, u)$ are predictable and

$$
\sup _{(\omega, t, x) \in \Omega \times \mathbb{R}_{+} \mathbb{R}^{d}}[|f(t, x, u)-f(t, x, v)|+|h(t, x, u)-h(t, x, v)|] \leq k|u-v|,
$$

for some positive constant $k$, a.s.

We recall that, for $\alpha \in(1,2), \mathcal{C}^{\alpha}$ is the space of continuously differentiable functions whose partial derivatives of first order are $\{\alpha\}$ - Hölder continuous, where $\alpha=[\alpha]+\{\alpha\},[\alpha]$ meaning the integer part of $\alpha$ (see [27]); $\mathcal{C}^{0,1}$ is the space of Lipschitz continuous functions.

In the proof of the next theorem we will use the following Remark 5.5 of [15]:

For any $u \in H_{p}^{n+2}, m \in[n, n+2]$ and $\varepsilon>0$, we have

$$
\begin{aligned}
\|u\|_{m, p} & \leq N\|u\|_{n+2, p}^{\theta}\|u\|_{n, p}^{1-\theta} \\
& \leq N \theta \varepsilon\|u\|_{n+2, p}+N(1-\theta) \varepsilon^{-\frac{\theta}{1-\theta}}\|u\|_{n, p},
\end{aligned}
$$

where $\theta=\frac{m-n}{2}$ and $N$ depends only on $d, n, m$ and $p$.

In the following theorem $\tau$ denotes a fixed stopping time with respect to the filtration $\left\{\mathcal{F}_{t}, t \geq 0\right\}$ defined in Section 2.

Theorem 6. Assume (H1), (H2), and that there exists $\eta \in\left(\frac{1}{2}, 1\right)$ such that

$$
\nu_{\eta, d}=\left\|R_{\eta, d}\right\|_{\mathcal{H}}^{2}<+\infty .
$$

We also suppose that, for some $p \in[2,+\infty)$ the following conditions are satisfied:

(a): $u_{0} \in L_{p}\left(\Omega, \mathcal{F}_{0}, H_{p}^{1-\eta-\frac{2}{p}}\right)$,

(b):

where

$$
I^{p}(\tau)=E\left[\int_{0}^{\tau} \mathrm{d} t\left(\|f(t, \cdot, 0)\|_{-1-\eta, p}^{p}+\|\bar{h}(t, \cdot, 0)\|_{p}^{p}\right)\right]<+\infty,
$$

$$
\bar{h}(t, x, 0):=\left\|R_{\eta, d}(x-\cdot) h(t, \cdot, 0)\right\|_{\mathcal{H}} .
$$

Then, in the space $\mathcal{H}_{p}^{1-\eta}(\tau)$, equation (8) with the initial condition $u_{0}$ and coefficients satisfying (H1), (H2) posseses a unique solution u. Moreover,

$$
\|u\|_{\mathcal{H}_{p}^{1-\eta}(\tau)} \leq C\left(I(\tau)+\left(E\left(\left\|u_{0}\right\|_{1-\eta-\frac{2}{p}}^{p}\right)^{\frac{1}{p}}\right)\right),
$$

where the constant $C$ depends on $\eta, d, \alpha, p, \delta, k$ and $\tau$.

In addition, if conditions (a), (b) are satisfied for any $p \geq 2$ then, the trajectories of $u$ belong to the space of Hölder continuous functions $\mathcal{C}^{\gamma_{1}, \gamma_{2}}\left([0, \tau] \times \mathbb{R}^{d}\right)$, a.s. with $\gamma_{1} \in\left(0, \frac{1-\eta}{2}\right), \gamma_{2} \in(0,1-\eta)$. 
Proof. The existence and uniqueness of solution will follow by applying Theorem 2 to

$$
\begin{gathered}
f(t, x, u):=b^{i}(t, x) u_{x^{i}}(t, x)+f(t, x, u(t, x)), \\
g^{k}(t, x, u):=h(t, x, u(t, x)) v_{k}(x),
\end{gathered}
$$

and by taking $n=-(1+\eta)$. In fact, we will check that the hypotheses (A1) and (A2) are satisfied.

Since $n \in\left(-2,-\frac{3}{2}\right)$, we shall consider as space $B^{|n|+\gamma}$, with $\gamma>0$ and $|n|+\gamma$ not an integer, the space $\mathcal{C}^{\alpha}\left(\mathbb{R}^{d}\right)$, with $\alpha \in(1+\eta, 2)$.

Set $\bar{f}(t, x, u)=b^{i}(t, x) u_{x^{i}}(t, x)+f(t, x, u(t, x))$; we have to check the following conditions for $n=-(1+\eta)$ (see Assumption (A2) before):

(1): For any $u \in H_{p}^{n+2},\{\bar{f}(t, \cdot, u), t \geq 0\}$ is a predictable process with values on $H_{p}^{n}$.

(2): $\bar{f}(\cdot, *, 0) \in \mathbb{H}_{p}^{n}$, a.s.

(3): $\bar{f}$ is a continuous function in $u$ a.s.

(4): For any $\varepsilon>0$, there exists $K_{\varepsilon}$ such that, for every $u, v \in H_{p}^{n+2}, t, \omega$,

$$
\|\bar{f}(t, \cdot, u)-\bar{f}(t, \cdot, v)\|_{n, p} \leq \varepsilon\|u-v\|_{n+2, p}+K_{\varepsilon}\|u-v\|_{n, p} .
$$

The predictability of $\bar{f}$ clearly follows from the same property of $b$ and $f$.

Let $u \in H_{p}^{n+2}$; then $u_{x^{i}} \in H_{p}^{n+1}$. Notice that, since $|n+1| \in\left(\frac{1}{2}, 1\right)$, the space $B^{|n+1|+\gamma}$ coincides with the space of the $\alpha$-Hölder continuous functions for some $\alpha \in(0,1)$. Since $\mathcal{C}^{0,1}\left(\mathbb{R}^{d}\right) \subset \mathcal{C}^{\alpha}\left(\mathbb{R}^{d}\right)$, Lemma 5.2 in Krylov [15] applied to $b$ and $u$ yields

$$
\left\|b^{i} u_{x^{i}}\right\|_{n+1, p} \leq\|b\|_{B^{|n+1|+\gamma}}\left\|u_{x^{i}}\right\|_{n+1, p}<+\infty .
$$

Thus $b^{i} u_{x^{i}} \in H_{p}^{n}$.

Moreover, $u \in L_{p}$ and the Lipschitz condition of $f$ with respect to $u$ implies $f(u)-f(0) \in L_{p} \subset H_{p}^{n}$. By (14), $f(t, \cdot, 0) \in H_{p}^{n}$ a.e. on $\rrbracket 0, \tau \rrbracket$. Therefore, (2) holds. In addition

$$
|f(t, \cdot, u)| \leq k|u|+|f(t, \cdot, 0)| .
$$

This proves $f(t, \cdot, u) \in H_{p}^{n}$ and thus $\bar{f}(t, \cdot, u) \in H_{p}^{n}$ as well.

Since $n<-1$, applying again Lemma 5.2 in [15], we have

$$
\begin{aligned}
\left\|b^{i} u_{x^{i}}\right\|_{n, p} & \leq\left\|b^{i} u_{x^{i}}\right\|_{-1, p} \leq\|b\|_{\mathcal{C}^{0,1}}\left\|u_{x^{i}}\right\|_{-1, p} \\
& \leq K\|u\|_{p}=K\|u\|_{n+2+\eta-1, p}
\end{aligned}
$$

where in the last identity we have used that $n+2+\eta-1=0$. This fact, together with the Lipschitz property of $f$ with respect to $u$, prove (3).

We also have

$$
\|f(t, \cdot, u)-f(t, \cdot, v)\|_{n, p} \leq\|f(t, \cdot, u)-f(t, \cdot, v)\|_{p} \leq K\|u-v\|_{p} .
$$

Then

where

$$
\|\bar{f}(t, \cdot, u)-\bar{f}(t, \cdot, v)\|_{n, p} \leq A+B
$$

by (19) and (18), respectively.

$$
\begin{gathered}
A=\|f(t, \cdot, u)-f(t, \cdot, v)\|_{n, p} \leq K\|u-v\|_{p}, \\
B=\left\|b^{i} u_{x^{i}}-b^{i} v_{x^{i}}\right\|_{n, p} \leq K\|u-v\|_{p},
\end{gathered}
$$

We now apply the above quoted Remark 5.5 of Krylov [15] to $m=n+2+\eta-1=0$. Notice that $\theta=-\frac{n}{2}>0$, $1-\theta=\frac{2+n}{2}>0,-\frac{\theta}{1-\theta}=\frac{n}{2+n}<0$. This yields property (4). 
Concerning the coefficient $g(t, x, u)=\left\{h\left(t, x, u(t, x) v_{k}(x)\right\}_{k \geq 0}\right.$ we have to check first of all that, for any $u \in H_{p}^{n+2},\{g(t, \cdot, u), t \geq 0\}$ is a predictable process with values on $H_{p}^{n+1}\left(l_{2}\right)$. This is a simple consequence of the fact that $h$ is predictable and $v_{k}(x)$ is deterministic. Moreover, since $h$ is Lipschitz, it is immediate also to prove that $g$ is a.s. continuous in $u$.

Let us now prove that $g(\cdot, *, 0) \in \mathbb{H}_{p}^{n+1}\left(\tau, l_{2}\right)$. Since $n+1=-\eta$, Lemma 3 yields

$$
\begin{aligned}
\|g(t, \cdot, u)\|_{n+1, p} & \leq\|g(t, \cdot, u)-g(t, \cdot, 0)\|_{n+1, p}+\|g(t, \cdot, 0)\|_{n+1, p} \\
& \leq\|h(t, \cdot, u)-h(t, \cdot, 0)\|_{p}+\|h(t, \cdot, 0)\|_{p} \\
& \leq\|u\|_{p}+\|h(t, \cdot, 0)\|_{p}<+\infty
\end{aligned}
$$

and

with $\bar{h}(t, \cdot, 0)$ defined in (15). Thus

$$
\|g(t, \cdot, 0)\|_{n+1, p}=\|\bar{h}(t, \cdot, 0)\|_{p}
$$

$$
\begin{aligned}
\|g(t, \cdot, 0)\|_{\mathbb{H}_{p}^{n+1}\left(\tau, l_{2}\right)}^{p} & =E\left(\int_{0}^{\tau}\|g(t, *, 0)\|_{n+1, p}^{p} \mathrm{~d} t\right) \\
& =E\left(\int_{0}^{\tau}\|\bar{h}(t, *, 0)\|_{p}^{p} \mathrm{~d} t\right)<+\infty,
\end{aligned}
$$

by (14) and we obtain $g(\cdot, *, 0) \in \mathbb{H}_{p}^{n+1}\left(\tau, l_{2}\right)$.

It remains to check that for any $\varepsilon>0$, there exists a constant $K_{\varepsilon}$ such that, for any $u, v \in H_{p}^{n+2}, t, \omega$,

$$
\|g(t, \cdot, u)-g(t, \cdot, v)\|_{n+1, p} \leq \varepsilon\|u-v\|_{n+2, p}+K_{\varepsilon}\|u-v\|_{n, p} .
$$

Applying again Lemma 3 and the Lipschitz property of $h$, yield

$$
\begin{aligned}
\|g(t, \cdot, u)-g(t, \cdot, v)\|_{n+1, p} & \leq C\|h(t, \cdot, u)-h(t, \cdot, v)\|_{p} \\
& \leq K\|u-v\|_{p}=K\|u-v\|_{n+2+\eta-1, p} .
\end{aligned}
$$

Then the above property follows, as for $\bar{f}$, from the above-mentioned Remark 5.5 in [15].

This finishes the proof of the existence and uniqueness of solution for equation (8) in the space $\mathcal{H}_{p}^{1-\eta}(\tau)$, and of the bound (16).

Let us now check the Hölder continuity of the trajectories of the solution, in a similar way as in Remark 8.7 in [15]. Let $p>2, \frac{1}{2}>\beta>\alpha>\frac{1}{p}$. Then by Theorem 7.2 in [15], a.s.

$$
u \in \mathcal{C}^{\alpha-1 / p}\left([0, \tau], H_{p}^{1-\eta-2 \beta}\right) .
$$

The space $H_{p}^{1-\eta-2 \beta}$ is embedded into $\mathcal{C}^{\gamma}\left(\mathbb{R}^{d}\right)$ for $\gamma<1-\eta-2 \beta-\frac{d}{p}$, whenever $1-\eta-2 \beta-\frac{d}{p}>0$ (see for instance Theorem E.12 of [26]). Thus, taking $p$ big enough and $\alpha, \beta$ small, we prove that $u$ is $\gamma_{2}$ Hölder continuous in $x$, with $\gamma_{2}<1-\eta$, uniformly in $t$. On the other hand, the conditions $\beta<\frac{1}{2}, 1-\eta-2 \beta-\frac{d}{p}>0$ are simultaneously satisfied for $p$ big enough whenever $\beta<\frac{1-\eta}{2} \wedge \frac{1}{2}=\frac{1-\eta}{2}$. Thus $u$ is Hölder continuous in $t$ of order $\gamma_{1}<\frac{1-\eta}{2}$, uniformly in $x$.

This finishes the proof of the theorem.

Remark 7. The assumptions of Theorem 6 ensuring Hölder continuity are satisfied if, for instance, $u_{0}(\omega, \cdot)$ is a.s. a $\mathcal{C}^{\infty}$ function with compact support and $f(t, x, 0)=h(t, x, 0)=0$. 


\section{Mild Formulation: Results on the existence AND Uniqueness of a solution}

In this section we consider the formal expression (8), but now, we assume that the coefficients $a, b$ are deterministic. More precisely, we fix a finite time horizon $T>0$ and we assume the following set of assumptions:

$\left(\mathbf{H 1}^{\prime}\right): a^{i, j}, b^{i}:[0, T] \times \mathbb{R}^{d} \rightarrow \mathbb{R}, i, j=1, \ldots, d$ are $\frac{\alpha}{2}$-Hölder continuous in $t \in[0, T], \alpha$-Hölder continuous in $x \in \mathbb{R}^{d}$, for some $\alpha \in(0,1)$. In addition, for any $\lambda \in \mathbb{R}^{d}$, there exist $K, \delta>0$ such that

$$
\delta|\lambda|^{2} \leq a^{i, j}(t, x) \lambda^{i} \lambda^{j} \leq K|\lambda|^{2} .
$$

$\left(\mathbf{H 2}^{\prime}\right): f, h: \Omega \times[0, T] \times \mathbb{R}^{d} \times \mathbb{R} \rightarrow \mathbb{R}$ are such that, for any $x \in \mathbb{R}^{d}$ and $u \in \mathbb{R}, f(\cdot, x, u), h(\cdot, x, u)$ are predictable processes satisfying the Lipschitz condition

$$
\sup _{(\omega, t, x) \in \Omega \times[0, T] \times \mathbb{R}^{d}}[|f(t, x, u)-f(t, x, v)|+|h(t, x, u)-h(t, x, v)|] \leq k|u-v|,
$$

for any $u, v \in \mathbb{R}$.

Following classical approaches on SPDEs, one can think of equation (8) with the initial condition $u(0, x)=u_{0}(x)$ as a stochastic Cauchy problem

$$
\left\{\begin{array}{l}
\mathcal{L} u(t, x)=f(t, x, u(t, x))+h(t, x, u(t, x)) F(\mathrm{~d} t, \mathrm{~d} x) \\
u(0, x)=u_{0}(x)
\end{array}\right.
$$

where $\mathcal{L}$ is the second order operator with coefficients depending on $t$ and $x$, acting on functions defined on $[0, T] \times \mathbb{R}^{d}$, given by

$$
\mathcal{L}=\frac{\partial}{\partial t}-\sum_{i, j=1}^{d} a^{i, j}(t, x) \partial_{x_{i} x_{j}}^{2}-\sum_{i=1}^{d} b^{i}(t, x) \partial_{x_{i}} .
$$

By virtue of $(20)$ the operator $\mathcal{L}$ is uniformly parabolic in $[0, T] \times \mathbb{R}^{d}$ (see [17], p. 11).

Let $G(t, x ; s, y)$ be the fundamental solution of $\mathcal{L} u=0$. $G$ is a function defined on $[0, T] \times \mathbb{R}^{d} \times[0, T] \times$ $\mathbb{R}^{d} \cap\{(s, t): 0 \leq s \leq t \leq T\}$. Under the above assumptions on the coefficients of $\mathcal{L}, G$ is continuous in all its variables and for any fixed $s \in[0, T], y \in \mathbb{R}^{d}, G(\cdot, *, ; s, y)$ is twice continuously differentiable in $x$, once continuously differentiable in $t$ and satisfies the estimates

$$
\left|\partial_{x}^{\mu} \partial_{t}^{\nu} G(t, x ; s, y)\right| \leq C(t-s)^{-\frac{d+|\mu|+2 \nu}{2}} \exp \left(-c \frac{|x-y|^{2}}{t-s}\right)
$$

where $\mu=\left(\mu_{1}, \ldots, \mu_{d}\right) \in \mathbb{N}^{d}, \nu \in \mathbb{N},|\mu|+2 \nu \leq 2$, with $|\mu|=\sum_{j=1}^{d} \mu_{j}$ (see (13.3), p. 376 in [17]). Moreover, $G$ is a positive function (Th. 11 in [9]).

Let us now introduce the notion of mild solution. A predictable stochastic process $\left\{u^{M}(t, x),(t, x) \in[0, T] \times\right.$ $\left.\mathbb{R}^{d}\right\}$ is said to be a mild solution to the stochastic Cauchy problem (21) if it satisfies the equation

$$
\begin{aligned}
u^{M}(t, x)= & \int_{\mathbb{R}^{d}} \mathrm{~d} y G(t, x ; 0, y) u_{0}(y) \\
& +\int_{0}^{t} \int_{\mathbb{R}^{d}} F(\mathrm{~d} s, \mathrm{~d} y) G(t, x ; s, y) h\left(s, y, u^{M}(s, y)\right) \\
& +\int_{0}^{t} \mathrm{~d} s \int_{\mathbb{R}^{d}} \mathrm{~d} y G(t, x ; s, y) f\left(s, y, u^{M}(s, y)\right) .
\end{aligned}
$$

Notice that, in order to give a rigourous meaning to equation (24), we must specify the space where the solution belongs to. 
Using the CONS $\left\{e_{k}, k \geq 0\right\}$ of $\mathcal{H}$ introduced in Section 2, the stochastic integral in (24) can also be written as

$$
\sum_{k=0}^{\infty} \int_{0}^{t} W^{k}(\mathrm{~d} s)\left\langle G(t, x ; s, \cdot) h\left(s, \cdot, u^{M}(s, \cdot)\right), e_{k}\right\rangle,
$$

with $W^{k}(t)=\int_{0}^{t} \int_{\mathbb{R}^{d}} F(\mathrm{~d} s, \mathrm{~d} y) e_{k}(y)$.

In the sequel, we denote by $G_{0}(t, x)$ the $d$-dimensional Gaussian density, zero mean, with variance $t \operatorname{Id}_{n}$. $\operatorname{The}$ inequality (23) implies

$$
|G(t, x ; s, y)|=G(t, x ; s, y) \leq C_{1} G_{0}\left(C_{2}(t-s),(x-y)\right),
$$

for some positive constants $C_{1}, C_{2}$.

Morevoer,

$$
\int_{0}^{t} \mathrm{~d} s \int_{\mathbb{R}^{d}} \Gamma(\mathrm{d} z)\left(G_{0}(s, x-\cdot) * G_{0}(s, x-\cdot)\right)(z)=\int_{0}^{t} \mathrm{~d} s \int_{\mathbb{R}^{d}} \mu(\mathrm{d} \xi)\left|\mathcal{F} G_{0}(t-s, \cdot)(\xi)\right|^{2} \leq C \int_{\mathbb{R}^{d}} \frac{\mu(\mathrm{d} \xi)}{1+|\xi|^{2}}<\infty .
$$

In order to compare mild and weak solutions, we need a theorem on existence and uniqueness of mild solution.

Theorem 8. Fix $p \in[2, \infty)$. Assume $\left(H 1^{\prime}\right),\left(H 2^{\prime}\right)$ and

$$
E\left[\int_{0}^{T} \mathrm{~d} s\left(\|h(s, \cdot, 0)\|_{p}^{p}+\|f(s, y, 0)\|_{p}^{p}\right)\right]<\infty .
$$

Suppose also that $u_{0} \in L_{p}\left(\mathbb{R}^{d}\right)$ and

$$
\int_{\mathbb{R}^{d}} \frac{\mu(\mathrm{d} \xi)}{1+|\xi|^{2}}<\infty
$$

Then, there exists a unique stochastic process $\left\{u^{M}(t, x),(t, x) \in[0, T] \times \mathbb{R}^{d}\right\}$ that belongs to $L_{p}\left(\Omega \times[0, T] ; L_{p}\left(\mathbb{R}^{d}\right)\right)$ and satisfies (24).

Proof. We shall divide the proof into two steps. First, we prove that the mapping defined on on $L_{p}(\Omega \times$ $\left.[0, T] ; L_{p}\left(\mathbb{R}^{d}\right)\right)$ by

$$
\begin{aligned}
\mathcal{T} u(t, x)= & \int_{\mathbb{R}^{d}} \mathrm{~d} y G(t, x ; 0, y) u_{0}(y) \\
& +\int_{0}^{t} \int_{\mathbb{R}^{d}} F(\mathrm{~d} s, \mathrm{~d} y) G(t, x ; s, y) h(s, y, u(s, y)) \\
& +\int_{0}^{t} \mathrm{~d} s \int_{\mathbb{R}^{d}} \mathrm{~d} y G(t, x ; s, y) f(s, y, u(s, y))
\end{aligned}
$$

takes values on the same space. Secondly, we check that this map is a contraction.

Step 1. Let $u \in L_{p}\left(\Omega \times[0, T] ; L_{p}\left(\mathbb{R}^{d}\right)\right)$; then,

$$
E\left(\int_{0}^{T} \mathrm{~d} t \int_{\mathbb{R}^{d}} \mathrm{~d} x|\mathcal{T} u(t, x)|^{p}\right) \leq C\left(T_{1}+T_{2}+T_{3}\right),
$$


with

$$
\begin{aligned}
& T_{1}=\int_{0}^{T} \mathrm{~d} t \int_{\mathbb{R}^{d}} \mathrm{~d} x\left|\int_{\mathbb{R}^{d}} \mathrm{~d} y G(t, x ; 0, y) u_{0}(y)\right|^{p}, \\
& T_{2}=\int_{0}^{T} \mathrm{~d} t \int_{\mathbb{R}^{d}} \mathrm{~d} x E\left(\left|\int_{0}^{t} \int_{\mathbb{R}^{d}} F(\mathrm{~d} s, \mathrm{~d} y) G(t, x ; s, y) h(s, y, u(s, y))\right|^{p}\right), \\
& T_{3}=\int_{0}^{T} \mathrm{~d} t \int_{\mathbb{R}^{d}} \mathrm{~d} x E\left(\left|\int_{0}^{t} \mathrm{~d} s \int_{\mathbb{R}^{d}} \mathrm{~d} y G(t, x ; s, y) f(s, y, u(s, y))\right|\right)^{p} .
\end{aligned}
$$

Hölder's inequality, the properties of $G$ and Fubini's theorem yield

$$
\begin{aligned}
T_{1} \leq & \int_{0}^{T} \mathrm{~d} t \int_{\mathbb{R}^{d}} \mathrm{~d} x\left(\int_{\mathbb{R}^{d}} \mathrm{~d} y G(t, x ; 0, y)\right)^{p-1} \int_{\mathbb{R}^{d}} \mathrm{~d} y G(t, x ; 0, y)\left|u_{0}(y)\right|^{p} \\
\leq & \sup _{(t, x) \in[0, T] \times \mathbb{R}^{d}}\left(\int_{\mathbb{R}^{d}} \mathrm{~d} y G(t, x ; 0, y)\right)^{p-1} \int_{0}^{T} \mathrm{~d} t \int_{\mathbb{R}^{d}} \mathrm{~d} y\left|u_{0}(y)\right|^{p} \\
& \times \int_{\mathbb{R}^{d}} \mathrm{~d} x G(t, x ; 0, y) \\
\leq & C \sup _{(t, x) \in[0, T] \times \mathbb{R}^{d}}\left(\int_{\mathbb{R}^{d}} \mathrm{~d} y G_{0}(t, x-y)\right)^{p}\left\|u_{0}\right\|_{p}^{p}<\infty .
\end{aligned}
$$

To deal with $T_{2}$, we apply Burkholder's inequality, then Hölder's inequality; we obtain

$$
\begin{aligned}
T_{2} \leq & C \int_{0}^{T} \mathrm{~d} t \int_{\mathbb{R}^{d}} \mathrm{~d} x E\left(\int_{0}^{t} \mathrm{~d} s \int_{\mathbb{R}^{d}} \Gamma(\mathrm{d} z) \int_{\mathbb{R}^{d}} \mathrm{~d} y G(t, x ; s, y) h(s, y, u(s, y))\right. \\
& \times G(t, x ; s, y-z) h(s, y-z, u(s, y-z)))^{\frac{p}{2}} \\
\leq & C \int_{0}^{T} \mathrm{~d} t \int_{\mathbb{R}^{d}} \mathrm{~d} x E\left(\int_{0}^{t} \mathrm{~d} s \int_{\mathbb{R}^{d}} \Gamma(\mathrm{d} z) \int_{\mathbb{R}^{d}} \mathrm{~d} y G_{0}(t-s, y-x)\right. \\
& \left.\times G_{0}(t-s, y-z-x)|h(s, y, u(s, y))||h(s, y-z, u(s, y-z))|\right)^{\frac{p}{2}} \\
\leq & C \int_{0}^{T} \mathrm{~d} t \int_{\mathbb{R}^{d}} \mathrm{~d} x\left(\int_{0}^{t} \mathrm{~d} s \int_{\mathbb{R}^{d}} \Gamma(\mathrm{d} z)\left(G_{0}(t-s, \cdot-x) * \tilde{G}_{0}(t-s, \cdot-x)\right)(z)\right)^{\frac{p}{2}-1} \\
& \times \int_{0}^{t} \mathrm{~d} s \int_{\mathbb{R}^{d}} \Gamma(\mathrm{d} z) \int_{\mathbb{R}^{d}} \mathrm{~d} y G_{0}(t-s, y-x) G_{0}(t-s, y-z-x) \\
\times & E\left(|h(s, y, u(s, y))|^{\frac{p}{2}}|h(s, y-z, u(s, y-z))|^{\frac{p}{2}}\right) .
\end{aligned}
$$

Then, owing to (25),

$$
\begin{aligned}
T_{2} \leq & C \int_{0}^{T} \mathrm{~d} t \int_{\mathbb{R}^{d}} \mathrm{~d} x \int_{0}^{t} \mathrm{~d} s \int_{\mathbb{R}^{d}} \Gamma(\mathrm{d} z) \int_{\mathbb{R}^{d}} \mathrm{~d} y G_{0}(t-s, y-x) G_{0}(t-s, y-z-x) \\
& \times E\left(|h(s, y, u(s, y))|^{\frac{p}{2}}|h(s, y-z, u(s, y-z))|^{\frac{p}{2}}\right) .
\end{aligned}
$$

Since the covariance functional is translation invariant, the last expression is bounded by

$$
\begin{aligned}
& C \int_{0}^{T} \mathrm{~d} t \int_{\mathbb{R}^{d}} \mathrm{~d} x \int_{0}^{t} \mathrm{~d} s \int_{\mathbb{R}^{d}} \Gamma(\mathrm{d} z) \int_{\mathbb{R}^{d}} \mathrm{~d} y G_{0}(t-s, y) G_{0}(t-s, y-z) \\
& \quad \times E\left(|h(s, y+x, u(s, y+x))|^{\frac{p}{2}}|h(s, y-z+x, u(s, y-z+x))|^{\frac{p}{2}}\right) .
\end{aligned}
$$


Applying Fubini's theorem and Schwarz inequality yields

$$
\begin{aligned}
T_{2} \leq & C \int_{0}^{T} \mathrm{~d} t \int_{\mathbb{R}^{d}} \mathrm{~d} x \int_{0}^{t} \mathrm{~d} s \int_{\mathbb{R}^{d}} \Gamma(\mathrm{d} z) \int_{\mathbb{R}^{d}} \mathrm{~d} y G_{0}(t-s, y) G_{0}(t-s, y-z) \\
& \times E\left(|h(s, y+x, u(s, y+x))|^{\frac{p}{2}}|h(s, y-z+x, u(s, y-z+x))|^{\frac{p}{2}}\right) \\
\leq & C \int_{0}^{T} \mathrm{~d} t \int_{0}^{t} \mathrm{~d} s \int_{\mathbb{R}^{d}} \Gamma(\mathrm{d} z) \int_{\mathbb{R}^{d}} \mathrm{~d} y G_{0}(t-s, y) G_{0}(t-s, y-z) \\
& \times E\left(\int_{\mathbb{R}^{d}} \mathrm{~d} x|h(s, y+x, u(s, y+x))|^{p}\right)^{\frac{1}{2}} \\
& \times E\left(\int_{\mathbb{R}^{d}} \mathrm{~d} x|h(s, y-z+x, u(s, y-z+x))|^{p}\right)^{\frac{1}{2}} \\
= & C \int_{0}^{T} \mathrm{~d} t \int_{0}^{t} \mathrm{~d} s E\left(\int_{\mathbb{R}^{d}} \mathrm{~d} x|h(s, x, u(s, x))|^{p}\right) \\
& \int_{\mathbb{R}^{d}} \Gamma(\mathrm{d} z)\left(G_{0}(t-s, \cdot) * \tilde{G}_{0}(t-s, \cdot)\right)(z)
\end{aligned}
$$

where the last identity holds by the translation invariance of Lebesgue measure.

The Lipschitz continuity of $h$ yields

$$
E\left(\int_{\mathbb{R}^{d}} \mathrm{~d} x \mid h\left(s, x,\left.u(s, x)\right|^{p}\right) \leq C E\left(\|u(s, \cdot)\|_{p}^{p}+\|h(s, \cdot, 0)\|_{p}^{p}\right) .\right.
$$

Hence, by (25) and (26),

$$
\begin{aligned}
T_{2} & \leq C_{1} \int_{0}^{T} \mathrm{~d} t \int_{0}^{t} \mathrm{~d} s E\left(\|u(s, \cdot)\|_{p}^{p}\right)+C_{2} \int_{0}^{T} \mathrm{~d} t \int_{0}^{t} \mathrm{~d} s E\left(\|h(s, \cdot, 0)\|_{p}^{p}\right) \\
& \leq C_{1}\|u\|_{L_{p}\left(\Omega \times[0, T] ; L_{p}\left(\mathbb{R}^{d}\right)\right)}+C_{3} .
\end{aligned}
$$

The analysis of $T_{3}$ is simpler. Indeed, Hölder's inequality implies

$$
\begin{aligned}
T_{3} \leq & \int_{0}^{T} \mathrm{~d} t \int_{\mathbb{R}^{d}} \mathrm{~d} x\left(\int_{0}^{t} \mathrm{~d} s \int_{\mathbb{R}^{d}} \mathrm{~d} y G(t, x ; s, y)\right)^{p-1} \\
& \times \int_{0}^{t} \mathrm{~d} s \int_{\mathbb{R}^{d}} \mathrm{~d} y G(t, x ; s, y) E\left(|f(s, y, u(s, y))|^{p}\right) \\
\leq & C \int_{0}^{T} \mathrm{~d} t \int_{\mathbb{R}^{d}} \mathrm{~d} x \int_{0}^{t} \mathrm{~d} s \int_{\mathbb{R}^{d}} \mathrm{~d} y G(t, x ; s, y) E\left(|f(s, y, u(s, y))|^{p}\right),
\end{aligned}
$$

since

$$
\sup _{0 \leq s \leq t \leq T, x \in \mathbb{R}^{d}} \int_{\mathbb{R}^{d}} \mathrm{~d} y G(t, x ; s, y)<\infty .
$$

Then, Fubini's theorem yields

$$
\begin{aligned}
T_{3} & \leq C \int_{0}^{T} \mathrm{~d} t \int_{0}^{t} \mathrm{~d} s \int_{\mathbb{R}^{d}} \mathrm{~d} y E\left(|f(s, y, u(s, y))|^{p}\right) \int_{\mathbb{R}^{d}} \mathrm{~d} x G(t, x ; s, y) \\
& \leq C \int_{0}^{T} \mathrm{~d} t \int_{0}^{t} \mathrm{~d} s \int_{\mathbb{R}^{d}} \mathrm{~d} y E\left(|f(s, y, u(s, y))|^{p}\right) .
\end{aligned}
$$


The estimate (31) with $h$ replaced by $f$ and (26) imply

$$
\begin{aligned}
T_{3} & \leq C \int_{0}^{T} \mathrm{~d} t \int_{0}^{t} \mathrm{~d} s\left\{E\left(\|u(s, \cdot)\|_{p}^{p}\right)+\|f(s, \cdot, 0)\|_{p}^{p}\right\} \\
& \leq C_{1}\|u\|_{L_{p}\left(\Omega \times[0, T] ; L_{p}\left(\mathbb{R}^{d}\right)\right)}+C_{4} .
\end{aligned}
$$

Then, (28), (32) and (34) give

$$
\|\mathcal{T} u\|_{L_{p}\left(\Omega \times[0, T] ; L_{p}\left(\mathbb{R}^{d}\right)\right)} \leq C_{1}\|u\|_{L_{p}\left(\Omega \times[0, T] ; L_{p}\left(\mathbb{R}^{d}\right)\right)}+C_{2}
$$

This completes the proof of Step 1.

Step 2. The mapping $\mathcal{T}$ has a unique fixed point in $L_{p}\left(\Omega \times[0, T] ; L_{p}\left(\mathbb{R}^{d}\right)\right)$.

Indeed, let $u_{1}, u_{2} \in L_{p}\left(\Omega \times[0, T] ; L_{p}\left(\mathbb{R}^{d}\right)\right)$. Proceeding as in Step 1 and by virtue of the Lipschitz property of $f$ and $h$, we obtain

$$
\left\|\mathcal{T} u_{1}-\mathcal{T} u_{2}\right\|_{L_{p}\left(\Omega \times[0, t] ; L_{p}\left(\mathbb{R}^{d}\right)\right)}^{p} \leq C_{1} \int_{0}^{t} \mathrm{~d} s\left\|u_{1}-u_{2}\right\|_{L_{p}\left(\Omega \times[0, s] ; L_{p}\left(\mathbb{R}^{d}\right)\right)}^{p}
$$

for any $0 \leq t \leq T$.

Consequently, for $N$ big enough, the $N$-th iterate of $\mathcal{T}$ is a contraction on $L_{p}\left(\Omega \times[0, T] ; L_{p}\left(\mathbb{R}^{d}\right)\right)$.

For any $p \in[2, \infty)$, let $\mathcal{B}_{p}$ be the Banach space of real-valued predictable processes such that

$$
\sup _{(t, x) \in[0, T] \times \mathbb{R}^{d}} E\left(|u(t, x)|^{p}\right)<\infty
$$

With arguments not very far from those applied in the proof of the preceding theorem, we can obtain the following result, which gives existence of a random field solution to (24). The details are left to the reader.

Theorem 9. Fix $p \in[2, \infty)$. Assume $\left(H 1^{\prime}\right),\left(H 2^{\prime}\right)$ and

$$
\int_{0}^{T} \mathrm{~d} s \sup _{y \in \mathbb{R}^{d}} E\left(|h(s, y, 0)|^{p}+|f(s, y, 0)|^{p}\right)<\infty .
$$

Suppose also that $\left\|u_{0}\right\|_{\infty}<C$ and moreover,

$$
\int_{\mathbb{R}^{d}} \frac{\mu(\mathrm{d} \xi)}{1+|\xi|^{2}}<\infty
$$

Then, there exists a unique stochastic processes $\left\{u^{M}(t, x),(t, x) \in[0, T] \times \mathbb{R}^{d}\right\}$ belonging to $\mathcal{B}_{p}$ and satisfying (24).

\section{Equivalence BetWeEn WeAK AND MiLd FORMUlations}

We devote this section to study the relationship between the notions of solution introduced previously, for some particular classes of spde's. As a consequence, we deduce path properties of the mild solution. We start by giving an equivalent weak formulation. Then, we compare the weak and mild formulation when the differential operator is self-adjoint and has non random coefficients. 
Let us consider equation (8) written in terms of the sequence $\left\{W^{k}, k \geq 0\right\}$ of independent Brownian motions, that is

$$
\begin{aligned}
\mathrm{d} u(t, x)= & {\left[a^{i, j}(t, x) u_{x^{i}, x^{j}}(t, x)+b^{i}(t, x) u_{x^{i}}(t, x)\right] } \\
& +f(t, x, u(t, x)) \mathrm{d} t+g^{k}(t, x, u(t, x)) W^{k}(\mathrm{~d} t),
\end{aligned}
$$

$t \in[0, T]$, with initial condition $u(0, \cdot)=u_{0}$.

We have proved in Theorem 6 the existence of a unique function-valued stochastic process $\{u(t), t \in[0, T]\}$ satisfying

$$
\begin{gathered}
(u(t, \cdot), \phi)=\left(u_{0}, \phi\right)+\int_{0}^{t} \mathrm{~d} s\left(a^{i, j}(s, \cdot) u_{x^{i}, x^{j}}(s, \cdot)+\left(b^{i}(s, \cdot) u_{x^{i}}(s, \cdot), \phi\right)\right. \\
+\int_{0}^{t} \mathrm{~d} s(f(s, \cdot, u(s, \cdot)), \phi)+\int_{0}^{t} W^{k}(\mathrm{~d} s)\left(g^{k}(s, \cdot, u(s, \cdot)), \phi\right)
\end{gathered}
$$

for all $\phi \in \mathcal{C}_{0}^{\infty}\left(\mathbb{R}^{d}\right)$, with the pairing $(\cdot, \cdot)$ given in $(5)$. We shall say that the process $u$ is a weak solution of equation (38).

The next proposition establishes the equivalence between testing against functions depending on $x$ and functions depending on $t$ and $x$ (see Th. 1 in [25] for a similar result in a different context). To fix the notation, denote by $\mathcal{C}_{t, x ; 0}^{1,2}$ the space of functions $f:[0, T] \times \mathbb{R}^{d} \rightarrow \mathbb{R}$ of class $\mathcal{C}^{1}$ in $t, \mathcal{C}^{2}$ in $x$, with compact support, and by $\mathcal{C}_{t, x ; \exp }^{1,2}$ a similar class of functions where the property of having compact support is replaced by the property of being rapidly decreasing (the functions and their derivatives).

Proposition 10. We assume that the assumptions of Theorem 6 are satisfied. The stochastic process $u$ is a weak solution if and only if for any function $\Phi \in \mathcal{C}_{t, x ; \exp }^{1,2}$, the following identity holds:

$$
\begin{aligned}
(u(t, \cdot), \Phi(t, \cdot))= & \left(u_{0}, \Phi(0, \cdot)\right)+\int_{0}^{t} \mathrm{~d} s\left(u(s, \cdot), \partial_{s} \Phi(s, \cdot)\right) \\
& +\int_{0}^{t} \mathrm{~d} s\left(a^{i, j}(s, \cdot) u_{x^{i}, x^{j}}(s, \cdot)+b^{i}(s, \cdot) u_{x^{i}}(s, \cdot), \Phi(s, \cdot)\right) \\
& +\int_{0}^{t} \mathrm{~d} s(f(s, \cdot, u(s, \cdot)), \Phi(s, \cdot))+\int_{0}^{t} W^{k}(\mathrm{~d} s)\left(g^{k}(s, \cdot, u(s, \cdot)), \Phi(s, \cdot)\right) .
\end{aligned}
$$

Proof. The "only if" part is trivial. To complete the proof, we proceed into three steps.

Step 1. Let us prove the result in the case where

$$
\Phi(t, x)=\varphi(t) \phi(x)
$$

with $\varphi \in \mathcal{C}^{1}([0, T])$ and $\phi \in \mathcal{C}_{0}^{2}\left(\mathbb{R}^{d}\right)$. 
In equation (38), we set $t=\sigma$, multiply each term by $\varphi^{\prime}(\sigma)$ and then integrate on $(0, t)$ with respect to $\sigma$. We obtain

$$
\begin{aligned}
\int_{0}^{t} \mathrm{~d} \sigma \varphi^{\prime}(\sigma)(u(\sigma, \cdot), \phi)= & \varphi(t)\left(u_{0}, \phi\right)-\varphi(0)\left(u_{0}, \phi\right) \\
& +\int_{0}^{t} \mathrm{~d} \sigma \varphi^{\prime}(\sigma) \int_{0}^{\sigma} \mathrm{d} s\left(a^{i, j}(s, \cdot) u_{x^{i}, x^{j}}(s, \cdot)+b^{i}(s, \cdot) u_{x^{i}}(s, \cdot), \phi\right) \\
& +\int_{0}^{t} \mathrm{~d} \sigma \varphi^{\prime}(\sigma) \int_{0}^{\sigma} \mathrm{d} s(f(s, \cdot, u(s, \cdot)), \phi) \\
& +\int_{0}^{t} \mathrm{~d} \sigma \varphi^{\prime}(\sigma) \int_{0}^{\sigma} W^{k}(\mathrm{~d} s)\left(g^{k}(s, \cdot, u(s, \cdot)), \phi\right) .
\end{aligned}
$$

Set

$$
\begin{aligned}
& I_{1}=\int_{0}^{t} \mathrm{~d} \sigma \varphi^{\prime}(\sigma) \int_{0}^{\sigma} \mathrm{d} s\left(a^{i, j}(s, \cdot) u_{x^{i}, x^{j}}(s, \cdot)+b^{i}(s, \cdot) u_{x^{i}}(s, \cdot), \phi\right), \\
& I_{2}=\int_{0}^{t} \mathrm{~d} \sigma \varphi^{\prime}(\sigma) \int_{0}^{\sigma} \mathrm{d} s(f(s, \cdot, u(s, \cdot)), \phi), \\
& I_{3}=\int_{0}^{t} \mathrm{~d} \sigma \varphi^{\prime}(\sigma) \int_{0}^{\sigma} W^{k}(\mathrm{~d} s)\left(g^{k}(s, \cdot, u(s, \cdot)), \phi\right) .
\end{aligned}
$$

Integrating by parts we obtain,

$$
\begin{aligned}
& I_{1}=I_{1}^{\prime}-\int_{0}^{t} \mathrm{~d} s \varphi(s)\left(a^{i, j}(s, \cdot) u_{x^{i}, x^{j}}(s, \cdot)+b^{i}(s, \cdot) u_{x^{i}}(s, \cdot), \phi\right), \\
& I_{2}=I_{2}^{\prime}-\int_{0}^{t} \mathrm{~d} s \varphi(s)(f(s, \cdot u(s, \cdot)), \phi), \\
& I_{3}=I_{3}^{\prime}-\int_{0}^{t} W^{k}(\mathrm{~d} s) \varphi(s)\left(g^{k}(s, \cdot, u(s, \cdot)), \phi\right),
\end{aligned}
$$

with

$$
\begin{aligned}
& I_{1}^{\prime}=\varphi(t) \int_{0}^{t} \mathrm{~d} s\left(a^{i, j}(s, \cdot) u_{x^{i}, x^{j}}(s, \cdot)+b^{i}(s, \cdot) u_{x^{i}}(s, \cdot), \phi\right) \\
& I_{2}^{\prime}=\varphi(t) \int_{0}^{t} \mathrm{~d} s(f(s, \cdot, u(s, \cdot)), \phi) \\
& I_{3}^{\prime}=\varphi(t) \int_{0}^{t} W^{k}(\mathrm{~d} s)\left(g^{k}(s, \cdot, u(s, \cdot)), \phi\right)
\end{aligned}
$$

Thus, noticing that

$$
\varphi(t)\left(u_{0}, \phi\right)+I_{1}^{\prime}+I_{2}^{\prime}+I_{3}^{\prime}=\varphi(t)(u(t, \cdot), \phi),
$$


we obtain

$$
\begin{aligned}
\int_{0}^{t} \mathrm{~d} \sigma\left(u(\sigma, \cdot), \partial_{\sigma} \Phi(\sigma, \cdot)\right)= & \int_{0}^{t} \mathrm{~d} \sigma \varphi^{\prime}(\sigma)(u(\sigma, \cdot), \phi) \\
= & \varphi(t)(u(t, \cdot), \phi)-\varphi(0)\left(u_{0}, \phi\right) \\
& -\left\{\int_{0}^{t} \mathrm{~d} \sigma \varphi(\sigma)\left(a^{i, j}(\sigma, \cdot) u_{x^{i}, x^{j}}(\sigma, \cdot)+b^{i}(\sigma, \cdot) u_{x^{i}}(\sigma, \cdot), \phi\right)\right. \\
& +\int_{0}^{t} \mathrm{~d} \sigma \varphi(\sigma)(f(\sigma, \cdot u(\sigma, \cdot)), \phi) \\
& \left.+\int_{0}^{t} W^{k}(\mathrm{~d} \sigma) \varphi(\sigma)\left(g^{k}(\sigma, \cdot, u(\sigma \cdot)), \phi\right)\right\}
\end{aligned}
$$

yielding (39).

Step 2. We now prove the result for $\Phi \in \mathcal{C}_{t, x, 0}^{1,2}$. For any compact set $\mathcal{K} \subset \mathbb{R}^{d}$ and any function $\Phi$ defined on $[0, T] \times \mathbb{R}^{d}$, set

$$
\|\Phi\|_{\mathcal{K}}=\sup _{(t, x) \in[0, T] \times \mathcal{K}}\left(|\Phi(t, x)|+\left|\partial_{t} \Phi(t, x)\right|+\sum_{|k| \leq 2}\left|\partial_{x}^{|k|} \Phi(t, x)\right|\right) .
$$

Fix $m \geq 1$. For any function $\Phi \in \mathcal{C}_{t, x, 0}^{1,2}$, there exists a polynomial

$$
p_{m}(t, x)=\sum_{\alpha, \beta \geq 0} c_{\alpha, \beta}^{(m)} x^{\alpha} t^{\beta},
$$

$\alpha=\left(\alpha_{1}, \ldots, \alpha_{d}\right)$, such that $\left\|\Phi-p_{m}\right\|_{\mathcal{K}}<\frac{1}{m}$, where we have assumed that the support of $\Phi$ is included in $[0, T] \times \mathcal{K}$ (see e.g. Kirillov and Gvishiani [12], p. 77). For simplicity, we will remove the subscript $\mathcal{K}$ in the norm $\|\cdot\| \mathcal{K}$.

We have proved in Step 1 that (39) holds with $\Phi:=p_{m}$. Set $\psi_{m}(t, x)=p_{m}(t, x)-\Phi(t, x)$. We now check that the $L^{1}(\Omega)$ norm of any term in (39), when $\Phi$ is replaced by $\psi_{m}(t, x)$, tends to 0 as $m$ tends to infinity. This shall finish the proof of the proposition.

Indeed, let us first prove that

$$
\lim _{m \rightarrow \infty} E\left(\left|\left(u_{0}, \psi_{m}(0, \cdot)\right)\right|\right)=0 .
$$

Hölder's inequality with $\frac{1}{p}+\frac{1}{q}=1$ yields

$$
\begin{aligned}
E\left(\left|\left(u_{0}, \psi_{m}(0, \cdot)\right)\right|\right) & \leq E\left(\int_{\mathbb{R}^{d}} \mathrm{~d} x\left|(1-\Delta)^{\frac{n_{0}}{2}} u_{0}(x)\right|\left|(1-\Delta)^{-\frac{n_{0}}{2}} \psi_{m}(0, x)\right|\right) \\
& \leq E\left(\left\|u_{0}\right\|_{n_{0}, p}\right)\left\|\psi_{m}(0, \cdot)\right\|_{-n_{0}, q} .
\end{aligned}
$$

Assume first $n_{0}:=1-\eta-\frac{2}{p} \geq 0$. Since,

$$
\left\|\psi_{m}(0, \cdot)\right\|_{-n_{0}, q} \leq C\left\|\psi_{m}(0, \cdot)\right\|_{q} \leq C\left\|\psi_{m}(0, \cdot)\right\|<\frac{C}{m}
$$

for any $q \in(1, \infty)$, the inequality (42) yields

$$
E\left(\left|\left(u_{0}, \psi_{m}(0, \cdot)\right)\right|\right) \leq \frac{C}{m}
$$

yielding (41). 
Assume that $n_{0}:=1-\eta-\frac{2}{p}<0$. The restrictions on $\eta$ and $p$ yield in this case $-n_{0} \in(0,1)$. Therefore,

$$
\begin{aligned}
\left\|\psi_{m}(0, \cdot)\right\|_{-n_{0}, q} & \leq C\left\|\psi_{m}(0, \cdot)\right\|_{2, q} \\
& \leq\left\|\psi_{m}(0, \cdot)\right\|_{q}+\left\|\Delta \psi_{m}(0, \cdot)\right\|_{q} \leq \frac{C}{m} .
\end{aligned}
$$

Then we obtain (41) from (42).

Set $m_{0}=1-\eta$. Notice that $m_{0}>0$. Then, as we did before for the case $n_{0} \geq 0$,

$$
E\left(\left|\int_{0}^{t} \mathrm{~d} s\left(u(s, \cdot), \partial_{s} \psi_{m}(s, \cdot)\right)\right|\right) \leq \int_{0}^{t} \mathrm{~d} s E\left(\|u(s, \cdot)\|_{m_{0}, p}\right)\left\|\partial_{s} \psi_{m}(s, \cdot)\right\| \|_{-m_{0}, q} \leq \frac{C}{m} .
$$

As in the proof of Theorem 6, we set $n=-(1+\eta)$. From Lemma 5.2 [15], it follows that

$$
E\left(\int_{0}^{t} \mathrm{~d} s\left(\left\|a^{i, j}(s, \cdot) u_{x^{i}, x^{j}}(s, \cdot)\right\|_{n, p}^{p}+\left\|b^{i}(s, \cdot) u_{x^{i}}(s, \cdot)\right\|_{n+1, p}^{p}\right)\right)<\infty .
$$

Then, since $n+1<0$ we obtain

$$
E\left(\left|\int_{0}^{t} \mathrm{~d} s\left(a^{i, j}(s, \cdot) u_{x^{i}, x^{j}}(s, \cdot)+b^{i}(s, \cdot) u_{x^{i}}(s, \cdot), \psi_{m}(s, \cdot)\right)\right|\right) \leq \frac{C}{m} .
$$

Following (19),

$$
\|f(s, \cdot, u(s, \cdot))\|_{n, p} \leq C\left(\|u\|_{p}+\|f(s, \cdot, 0)\|_{n, p}\right) .
$$

Consequently, since $-n \in\left(\frac{3}{2}, 2\right)$, using (14) we have

$$
E\left(\mid \int_{0}^{t} \mathrm{~d} s\left(f(s, \cdot, u(s, \cdot)), \psi_{m}(s, \cdot) \mid\right)\right) \leq \int_{0}^{t} \mathrm{~d} s E\left(\|f(s, \cdot, u(s, \cdot))\|_{n, p}\right)\left\|\psi_{m}(s, \cdot)\right\|_{-n, q} \leq \frac{C}{m} .
$$

We now deal with the stochastic integral by considering the $L^{2}(\Omega)$-norm. The isometry property of the stochastic integral yields,

$$
E\left|\sum_{k=1}^{\infty} \int_{0}^{t} \mathrm{~d} W_{s}^{k}\left(g^{k}(s, \cdot, u(s, \cdot)), \psi_{m}(s, \cdot)\right)\right|^{2}=\sum_{k=1}^{\infty} E \int_{0}^{t} \mathrm{~d} s\left(g^{k}(s, \cdot, u(s, \cdot)), \psi_{m}(s, \cdot)\right)^{2} .
$$

Using Schwarz's and Hölder's inequalities, this last expression is bounded by

$$
\sup _{s \in[0, T]}\left\|(1-\Delta)^{-\frac{\eta}{2}} \psi_{m}(s, \cdot)\right\|_{1}\left\|(1-\Delta)^{-\frac{\eta}{2}} \psi_{m}(s, \cdot)\right\|_{q} \int_{0}^{t} \mathrm{~d} s\left\|\left(\sum_{k=1}^{\infty}\left|(1-\Delta)^{-\frac{\eta}{2}} g^{k}(s, \cdot, u(s, \cdot))\right|^{2}\right)^{\frac{1}{2}}\right\|_{p}^{2},
$$

(see [15], p. 192). Hence,

$$
E\left|\sum_{k=1}^{\infty} \int_{0}^{t} \mathrm{~d} W_{s}^{k}\left(g^{k}(s, \cdot, u(s, \cdot)), \psi_{m}(s, \cdot)\right)\right|^{2} \leq \frac{C}{m} .
$$

With the estimates (41)-(46), we finish the proof of this step.

Step 3. Having established the validity of (39) for functions $\Phi \in \mathcal{C}_{t, x, 0}^{1,2}$, we finally prove it for functions $\Phi \in \mathcal{C}_{t, x, \exp }^{1,2}$. 
For any non-negative integer $r$, set

$$
\|\Phi\|_{(r)}=\sup _{(t, x) \in[0, T] \times \mathbb{R}^{d}}(1+|x|)^{r}\left(|\Phi(t, x)|+\left|\partial_{t} \Phi(t, x)\right|+\sum_{|k| \leq 2}\left|\partial_{x}^{|k|} \Phi(t, x)\right|\right) .
$$

Fix $r$ and $m$ non-negative integers. Following the same proof as that of Theorem III, Chapter VII in [22], we can find a function $\Phi_{m} \in \mathcal{C}_{t, x, 0}^{1,2}$ such that $\left\|\Phi-\Phi_{m}\right\|_{(r)} \leq \frac{1}{m}$.

For any $q \in[1, \infty), r>d$, and any real function $\psi$ defined on $\mathbb{R}^{d}$, we have

$$
\|\psi\|_{q} \leq C \sup _{x \in \mathbb{R}^{d}}\left((1+|x|)^{\frac{r}{q}}|\psi(x)|\right) .
$$

Indeed, $\int_{\mathbb{R}^{d}} \frac{\mathrm{d} x}{(1+|x|)^{r}}<\infty$.

Hence, the arguments in the proof of Step 2 remain valid for $\psi_{m}:=\Phi-\Phi_{m}$, substituting the norm $\|\cdot\|$ by $\|\cdot\|_{(r)}$ given in (47) and using (48).

This finishes the proof of the proposition.

We want now to prove that if a process $\left\{u^{W}(t, x),(t, x) \in[0, T] \times \mathbb{R}^{d}\right\}$ satisfies the weak formulation in the sense of (39) then, it also satisfies the mild formulation. To obtain this result, we restrict the class of operators. More precisely, we assume that $\mathcal{L}$ given in (22) is self-adjoint and also the following conditions on the coefficients:

$\left(\mathbf{H} 1^{\prime \prime}\right): a^{i, j}, b^{i}, \partial_{x_{k}} a^{i, j}, \partial_{x_{k}, x_{l}}^{2} a^{i, j}, \partial_{x_{k}} b^{i}:[0, T] \times \mathbb{R}^{d} \rightarrow \mathbb{R}, i, j, k, l=1, \ldots, d$, are bounded functions, $\frac{\alpha}{2}-$ Hölder continuous in $t \in[0, T]$ and $\alpha$-Hölder continuous in $x \in \mathbb{R}^{d}$, for some $\alpha \in(0,1)$. In addition, for any $\lambda \in \mathbb{R}^{d}$, there exist $K, \delta>0$ such that

$$
\delta|\lambda|^{2} \leq \sum_{i, j=1}^{d} a^{i, j}(t, x) \lambda^{i} \lambda^{j} \leq K|\lambda|^{2} .
$$

Notice that $\left(\mathrm{H}^{\prime \prime}\right)$ implies that the coefficients of the operator $\mathcal{L}$ given in $(22)$ satisfies the asumption (H1) of Theorem 6 .

Let $\mathcal{L}^{*}$ be the adjoint operator of $\mathcal{L}$ (see [9], p. 26), that is,

$$
\mathcal{L}_{t, x}^{*} u(t, x)=-\frac{\partial}{\partial t}-\sum_{i, j=1}^{d} \partial_{x^{i}, x^{j}}^{2}\left(a^{i, j}(t, x) u(t, x)\right)+\sum_{i=1}^{d} \partial_{x_{i}}\left(b^{i}(y, x) u(t, x)\right) .
$$

Under assumption (H1'), for every fixed $t \in[0, T], y \in \mathbb{R}^{d}$,

$$
\mathcal{L}_{s, x}^{*} G(t, y ; s, x)=0
$$

(Th. 15 in [9]). $\mathbb{R}$ by

Consider a function $v \in \mathcal{C}_{0}^{\infty}\left(\mathbb{R}^{d}\right)$, with compact support $\mathcal{K} \subset \mathbb{R}^{d}$. Fix $t \in(0, T]$ and define $v^{t}:[0, t] \times \mathbb{R}^{d} \longrightarrow$

$$
v^{t}(s, x)= \begin{cases}v(x), & \text { if } s=t, \\ \int_{\mathbb{R}^{d}}^{\mathrm{d} y} v(y) G(t, x ; s, y), & \text { if } s<t,\end{cases}
$$

$x \in \mathbb{R}^{d}$.

Using (49) it is not difficult to check that $v^{t}(s, x)$ belongs to $\mathcal{C}_{t, x, \exp }^{1,2}$.

Let us now prove the following auxiliary result. 
Lemma 11. Let $\left\{u^{W}(t, x),(t, x) \in[0, T] \times \mathbb{R}^{d}\right\}$ be a process satisfying the weak formulation given in (39). Let $v^{t}(s, x)$ be the function defined before. Then, the following identity holds

$$
\begin{aligned}
\left(u^{W}(t, \cdot), v\right)= & \left(u_{0}, v^{t}(0, \cdot)\right)+\int_{0}^{t} \mathrm{~d} s\left(f\left(s, \cdot, u^{W}(s, \cdot)\right), v^{t}(s, \cdot)\right) \\
& +\int_{0}^{t} W^{k}(\mathrm{~d} s)\left(g^{k}\left(s, \cdot, u^{W}(s, \cdot)\right), v^{t}(s, \cdot)\right) .
\end{aligned}
$$

Proof. Since $v^{t} \in \mathcal{C}_{t, x, \exp }^{1,2},(39)$ holds with $\Phi=v^{t}$. Proposition 10 tell us that proving (51) is equivalent to check

$$
\begin{aligned}
0= & \int_{0}^{t} \mathrm{~d} s\left(u^{W}(s, \cdot), \partial_{s} v^{t}(s, \cdot)\right) \\
& +\int_{0}^{t} \mathrm{~d} s\left(a^{i, j}(s, \cdot) u_{x^{i}, x^{j}}^{W}(s, \cdot)+b^{i}(s, \cdot) u_{x^{i}}^{W}(s, \cdot), v^{t}(s, \cdot)\right) \\
= & \int_{0}^{t} \mathrm{~d} s\left(u^{W}(s, \cdot), \partial_{s} v^{t}(s, \cdot)\right) \\
& +\int_{0}^{t} \mathrm{~d} s\left(u^{W}(s, \cdot), \partial_{x ?, x^{j}}^{2}\left(a^{i, j}(s, \cdot) v^{t}(s, \cdot)\right)-\partial_{x^{i}}\left(b^{i}(s, \cdot) v^{t}(s, \cdot)\right) .\right.
\end{aligned}
$$

By the definition of $v^{t}$ this reads

$$
0=\int_{\mathbb{R}^{d}} \mathrm{~d} y v(y) \int_{0}^{t} \mathrm{~d} s\left(u^{W}(s, \cdot), \mathcal{L}_{s, \cdot}^{*} G(t, \cdot ; s, y)\right) .
$$

Since $\mathcal{L}$ is a self-adjoint operator, $G$ is symmetric in $(x, y)$. Consequently $\mathcal{L}_{s, \cdot}^{*} G(t, \cdot ; s, y)=\mathcal{L}_{s, \cdot}^{*} G(t, y ; s, \cdot)=0$ for any $y \in \mathbb{R}^{d}, t>s$. This clearly implies (53).

Proposition 12. We assume that the above hypothesis (H1') and (H2) of Theorem 6 are satisfied. Let $\left\{u^{W}(t, x),(t, x) \in[0, T] \times \mathbb{R}^{d}\right\}$ be a weak solution in the sense of Proposition 10. Then, for each fixed $t \in[0, T]$ and any $x$-a.e.

$$
\begin{aligned}
u^{W}(t, x)= & \left(u_{0}, G(t, x ; 0, \cdot)\right)+\int_{0}^{t} \mathrm{~d} s\left(f\left(s, \cdot u^{W}(s, \cdot)\right), G(t, x ; s, \cdot)\right) \\
& +\int_{0}^{t} W^{k}(\mathrm{~d} s)\left(g^{k}\left(s, \cdot u^{W}(s, \cdot)\right), G(t, x ; s, \cdot)\right) .
\end{aligned}
$$

Proof. For a fixed $t \in[0, T]$, we write the expression (39) with $\Phi(s, x)=v^{t}(s, x)$, defined in (50). By virtue of Lemma 11 we obtain

$$
\begin{aligned}
\left(u^{W}(t, \cdot), v\right)= & \left(u_{0}, \int_{\mathbb{R}^{d}} \mathrm{~d} y v(y) G(t, \cdot ; 0, y)\right)+\int_{0}^{t} \mathrm{~d} s\left(f\left(s, \cdot, u^{W}(s, \cdot)\right), \int_{\mathbb{R}^{d}} \mathrm{~d} y v(y) G(t, \cdot ; s, y)\right) \\
& +\int_{0}^{t} W^{k}(\mathrm{~d} s)\left(g^{k}\left(s, \cdot, u^{W}(s, \cdot)\right), \int_{\mathbb{R}^{d}} \mathrm{~d} y v(y) G(t, \cdot ; s, y)\right) .
\end{aligned}
$$

Fubini's theorem implies

$$
\begin{aligned}
\left(u^{W}(t, \cdot), v\right)= & \int_{\mathbb{R}^{d}} \mathrm{~d} y v(y)\left[\left(u_{0}, G(t \cdot ; 0, y)\right)+\int_{0}^{t} \mathrm{~d} s\left(f\left(s, \cdot, u^{W}(s, \cdot)\right), G(t, \cdot ; s, y)\right)\right. \\
& \left.+\int_{0}^{t} W^{k}(\mathrm{~d} s)\left(g^{k}\left(s, \cdot u^{W}(s, \cdot)\right), G(t, \cdot ; s, y)\right)\right]
\end{aligned}
$$


Consequently, for any $x \in \mathcal{K}$, a.e. with respect to Lebesgue measure,

$$
\begin{aligned}
u^{W}(t, x)= & \left(u_{0}, G(t, \cdot ; 0, x)\right)+\int_{0}^{t} \mathrm{~d} s\left(f\left(s, \cdot, u^{W}(s, \cdot)\right), G(t, \cdot ; s, x)\right) \\
& +\int_{0}^{t} W^{k}(\mathrm{~d} s)\left(g^{k}\left(s, \cdot, u^{W}(s, \cdot)\right), G(t, \cdot ; s, x)\right) .
\end{aligned}
$$

Since $G(t, x ; s, y)=G(t, y ; s, x)$, for any $s \leq t$ and $x, y \in \mathbb{R}^{d}$, and $\mathcal{K}$ is arbitrary, this is equivalent to (54).

The next result, which is the main conclusion of this section, states that, if there exists a function-valued solution in the weak sense, then it must coincide with the mild solution. We need simultaneously the validity of the assumptions of Theorems 6 and 8 and Proposition 12. More precisely, we have the following theorem.

Theorem 13. Suppose that

(1) The operator $\mathcal{L}$ defined in (22) is self-adjoint and its coefficients are deterministic.

(2) The functions $a^{i, j}, b^{i}, \partial_{x_{k}} a^{i, j}, \partial_{x_{k}, x_{l}}^{2} a^{i, j}, \partial_{x_{k}} b^{i}:[0, T] \times \mathbb{R}^{d} \rightarrow \mathbb{R}, i, j, k, l=1, \ldots, d$, are bounded, $\frac{\alpha}{2}$ Hölder continuous in $t \in[0, T]$ and $\alpha$-Hölder continuous in $x \in \mathbb{R}^{d}$, for some $\alpha \in(0,1)$.

(3) For any $\lambda \in \mathbb{R}^{d}$, there exist $K, \delta>0$ such that

$$
\delta|\lambda|^{2} \leq \sum_{i, j=1}^{d} a^{i, j}(t, x) \lambda^{i} \lambda^{j} \leq K|\lambda|^{2} .
$$

(4) The coefficients of Equation (8) are predictable processes

$$
f, h: \Omega \times[0, T] \times \mathbb{R}^{d} \times \mathbb{R} \rightarrow \mathbb{R}
$$

such that the following conditions hold:

(a) For any $u, v \in \mathbb{R}$,

$$
\sup _{(\omega, t, x) \in \Omega \times[0, T] \times \mathbb{R}^{d}}\{|f(t, x, u)-f(t, x, v)|+|h(t, x, u)-h(t, x, v)|\} \leq k|u-v| .
$$

(b) For some fixed $p \in[2, \infty)$,

$$
E\left(\int_{0}^{T} \mathrm{~d} s\left(\|h(s, \cdot, 0)\|_{p}^{p}+\|f(s, \cdot, 0)\|_{p}^{p}\right)\right)<\infty .
$$

(5) There exists $\eta \in\left(\frac{1}{2}, 1\right)$ such that

$$
\int_{\mathbb{R}^{d}} \frac{\mu(\mathrm{d} \xi)}{\left(1+|\xi|^{2}\right)^{\eta}}<\infty
$$

(6) $u_{0} \in L_{p}\left(\mathbb{R}^{d}\right) \cap H_{p}^{1-\eta-\frac{2}{p}}$.

Then $u^{W}=u^{M}$ as processes in $L_{p}\left(\Omega \times[0, T] ; L_{p}\left(\mathbb{R}^{d}\right)\right)$. Consequently, $\omega$-a.s., $u^{W}(t, x)=u^{M}(t, x)$, a.e. with respect to Lebesgue measure on $[0, T] \times \mathbb{R}^{d}$. 


\section{Remark 14.}

(1) The above hypothesis 5 implies that $\left\|R_{\eta, d}\right\|_{\mathcal{H}}<\infty$.

Under this condition, we have proved in Lemma 3, that $\|\bar{h}\|_{p} \leq C\|h\|_{p}$, where $\bar{h}(x)=\left\|R_{\eta, d}(x-\cdot) h\right\|_{\mathcal{H}}$. Therefore the assumption 4 (b) implies

$$
E \int_{0}^{T} \mathrm{~d} s\|\bar{h}(s, \cdot, 0)\|_{p}^{p}<\infty
$$

(see (14) in Th. 6).

(2) From the relation $\|\cdot\|_{n, p} \leq\|\cdot\|_{m, p}, n \leq m$, it follows trivially that $\|\cdot\|_{-1-\eta, p} \leq\|\cdot\|_{p}$. Thus, assumption 4 (b) implies

$$
E \int_{0}^{T} \mathrm{~d} s\|f(s, \cdot, 0)\|_{-1-\eta, p}^{p}<\infty
$$

(see again (14) in Th. 6).

(3) The above remarks show that the assumptions of Theorem 6 and of Theorem 8 are fulfilled. Hence, the existence of $u^{W}$ satisfying the weak formulation of equation (8) and $u^{M}$ satisfying the mild formulation is assured.

Notice that

$$
\mathcal{H}_{p}^{1-\eta}(T) \subset L_{p}\left(\Omega \times[0, T] ; L_{p}\left(\mathbb{R}^{d}\right)\right) .
$$

Proof of Theorem 13. Equation (54) can be now written as

$$
\begin{aligned}
u^{W}(t, x)= & \int_{\mathbb{R}^{d}} \mathrm{~d} y u_{0}(y) G(t, x ; 0, y) \\
& +\int_{0}^{t} \mathrm{~d} s \int_{\mathbb{R}^{d}} \mathrm{~d} y f\left(s, y, u^{W}(s, y) G(t, x ; s, y)\right) \\
& +\int_{0}^{t} W^{k}(\mathrm{~d} s) \int_{\mathbb{R}^{d}} \mathrm{~d} y g^{k}\left(s, y, u^{W}(s, y) G(t, x ; s, y)\right) .
\end{aligned}
$$

We next prove that

$$
E\left(\int_{0}^{T} \mathrm{~d} t\left\|u^{W}(t, \cdot)-u^{M}(t, \cdot)\right\|_{p}^{p}\right)=0
$$

Indeed, from the equations satisfied by $u^{W}$ and $u^{M}$, respectively, it follows that

$$
E\left(\int_{0}^{T} \mathrm{~d} t\left\|u^{W}(t, \cdot)-u^{M}(t, \cdot)\right\|_{p}^{p}\right) \leq C\left(R_{1}+R_{2}\right),
$$

with

$$
\begin{aligned}
R_{1}= & E \int_{0}^{T} \mathrm{~d} t \int_{\mathbb{R}^{d}} \mathrm{~d} x \mid \int_{0}^{t} \mathrm{~d} s \int_{\mathbb{R}^{d}} \mathrm{~d} y\left(f\left(s, y, u^{W}(t, y)\right)-f\left(s, y, u^{M}(t, y)\right)\right) \\
& \times\left. G(t, x ; s, y)\right|^{p}, \\
R_{2}= & E \int_{0}^{T} \mathrm{~d} t \int_{\mathbb{R}^{d}} \mathrm{~d} x \mid \int_{0}^{t} W^{k}(\mathrm{~d} s) \int_{\mathbb{R}^{d}} \mathrm{~d} y\left(g^{k}\left(s, y, u^{W}(t, y)\right)-g^{k}\left(s, y, u^{M}(t, y)\right)\right) \\
& \times\left. G(t, x ; s, y)\right|^{p} .
\end{aligned}
$$


Following the arguments of the proof of Theorem 8 and by virtue of the Lipschitz assumptions on $f$ and $h$, we obtain

$$
R_{1}+R_{2} \leq C \int_{0}^{T} \mathrm{~d} t \int_{0}^{t} \mathrm{~d} s E\left(\int_{\mathbb{R}^{d}} \mathrm{~d} x\left|u^{W}(s, x)-u^{M}(s, x)\right|^{p}\right) .
$$

We conclude by Gronwall's lemma applied to the function

$$
\Psi(T)=E\left(\int_{0}^{T} \mathrm{~d} t\left\|u^{W}(t, \cdot)-u^{M}(t, \cdot)\right\|_{p}^{p}\right)
$$

for $T \geq 0$.

The conclusion of Theorem 13 can be strengthened assuming, for instance, instead of $4(\mathrm{~b})$, that $f(t, x, 0)=$ $h(t, x, 0)=0$ and $u_{0} \in \cap_{p \geq 2} L_{p}\left(\mathbb{R}^{d}\right)$. Indeed, in this case it has been proved that a.s.

$$
u^{W} \in \mathcal{C}^{\gamma_{1}, \gamma_{2}}\left([0, T] \times \mathbb{R}^{d}\right),
$$

with $\gamma_{1}<\frac{1-\eta}{2}, \gamma_{2}<1-\eta$, and we can show that $\omega$-a.s. $u^{M}$ owns the same property.

Acknowledgements. The first author wishes to thank the Institut de Matemàtica, Universitat de Barcelona and the Centre de Recerca Matemàtica (Bellaterra, Spain) for their support and warm hospitality. The second author expresses her thanks to the Università degli Studi di Padova for a kind invitation on July 2003.

\section{REFERENCES}

[1] M. Abramowitz and I.A. Stegun, Handbook of mathematical functions. National Bureau of Standards (1964).

[2] R.A. Adams, Sobolev spaces. Academic Press, New York-London (1975).

[3] E. Alòs, J.A. León and D. Nualart, Stochastic heat equation with random coefficients. Probab. Theory Related Fields 115 (1999) 41-94.

[4] R.C. Dalang and N.E. Frangos, The stochastic wave equation in two spatial dimensions. Ann. Probab. 26 (1998) $187-212$.

[5] R.C. Dalang, Extending the martingale measure stochastic integral with applications to spatially homogeneous s.p.d.e.'s. Electron. J. Probab. 4 (1999) 1-29.

[6] G. Da Prato and J. Zabczyk, Stochastic Equations in Infinite Dimensions, 2nd Edition. Cambridge University Press (1998).

[7] W.F. Donoghue, Distributions and Fourier transforms. Academic Press, New York (1969).

[8] S.D. Eidelman and N.V. Zhitarashu, Parabolic Boundary Value Problems. Birkhäuser Verlag, Basel (1998).

[9] A. Friedman, Partial differential equations of parabolic type. Prentice-Hall, Inc., Englewood Cliffs, N.J. (1964).

[10] I.M. Gel'fand and N.Ya. Vilenkin, Generalized functions. Vol. 4: Applications of harmonic analysis. Academic Press, New York (1964).

[11] M.A Krasnoselskii, E.I. Pustylnik, P.E. Sobolevski and P.P. Zabrejko, Integral operators in spaces of summable functions. Noordhoff International Publishing, Leyden (1976).

[12] A.A. Kirillov and A.D. Gvishiani, Theorems and problems in functional analysis. Springer-Verlag, New York-Berlin (1982).

[13] N.V. Krylov and B.L. Rozovsky, Stochastic evolution systems. Russian Math. Surveys 37 (1982) 81-105.

[14] N.V. Krylov, On $L_{p}$-theory of stochastic partial differential equations in the whole space. SIAM J. Math. Anal. 27 (1996) 313-340.

[15] N.V. Krylov, An analytic approach to SPDEs, in Stochastic partial differential equations: six perspectives, Math. Surveys Monogr. 64, American Mathematical Society, Providence (1999) 185-242.

[16] N.V. Krylov and V. Lototsky, A Sobolev space theory of SPDEs with constant coefficients on a half line. SIAM J. Math. Anal. 30 (1998) 298-325.

[17] O.A. Ladyženskaja, V.A. Solonnikov and N.N. Ural'ceva, Linear and Quasilinear Equations of Parabolic Type. Translations of Mathematical Monographs 23, American Mathematical Society (1968).

[18] O. Lévêque, Hyperbolic stochastic partial differential equations driven by boundary noises. Thèse 2452, Lausanne, EPFL (2001).

[19] R. Mikulevicius, On the Cauchy problem for parabolic SPDEs in Hölder classes. Ann. Probab. 28 (2000) 74-103.

[20] E. Pardoux, Stochastic partial differential equations and filtering of diffusion processes. Stochastics 3 (1979) $127-167$. 
[21] B.L. Rozovsky, Stochastic evolution equations. Linear theory and applications to non-linear filtering. Kluwer (1990).

[22] L. Schwartz, Théorie des distributions. Hermann, Paris (1966).

[23] M. Sanz-Solé and M. Sarrà, Path properties of a class of Gaussian processes with applications to spde's. Canadian Mathematical Society Conference Proceedings 28 (2000) 303-316.

[24] M. Sanz-Solé and M. Sarrà, Hölder Continuity for the stochastic heat equation with spatially correlated noise, in Progress in Probability 52, Birkhäuser Verlag (2002) 259-268.

[25] M. Sanz-Solé and P.-A. Vuillermot, Equivalence and Hölder-Sobolev regularity of solutions for a class of non-autonomous stochastic partial differential equations. Ann. Inst. H. Poincaré Probab. Statist. 39 (2003) 703-742.

[26] N. Shimakura, Partial differential operators of elliptic type. American Mathematical Society, Providence (1992).

[27] H. Triebel, Theory of function spaces. II. Monographs in Mathematics 84, Birkhäuser Verlag, Basel (1992).

[28] J.B. Walsh, An Introduction to Stochastic Partial Differential Equations, in École d'été de Probabilités de Saint-Flour XIV (1984). Lect. Notes Math. 1180 (1986) 265-439. 